\title{
ÉCOLOGIE D'Un TORRENT PYRÉNÉEN DE HAUTE MONTAGNE II. - CARACTÉRistiques ChimiQues
}

\author{
par P. Lavandier ${ }^{1}$ et C. MuR ${ }^{2}$.
}

Les caractéristiques chimiques du torrent d'Estaragne et de ses affluents, sont étudiées en relation avec les conditions géographiques générales.

Les bicarbonates et le calcium forment plus de $90 \%$ du contenu ionique des eaux. Les teneurs en fer total, $\mathrm{Ca}^{++}, \mathrm{HCO}_{3}^{-}, \mathrm{SO}_{4}^{--}, \mathrm{PO}_{4}-\mathrm{P}, \mathrm{NO}_{3}-\mathrm{N}, \mathrm{Mg}^{++}, \mathrm{K}^{+}$, diminuent entre l'amont et l'aval. Les concentrations en $\mathrm{Na}^{+}, \mathrm{Cl}^{-}$et $\mathrm{SiO}$, restent constantes ou s'accroissent légèrement le long du cours. La conductivité passe de $114 \mu \mathrm{mhos}$ à $80 \mu \mathrm{mhos}$, entre la source et l'embouchure. L'évolution de la composition chimique de l'eau est liée au passage de roches altérables et nues, à des roches cristallines couvertes de pelouse.

La plupart des caractéristiques présentent des corrélations significatives avec Ies débits. Ces relations ont permis d'évaluer - avec des limites de confiance les quantités de matéricl transporté. Lors des deux années d'étude, le torrent a déversé en moyenne $470 \mathrm{t}$ /an d'éléments dissous, dans le lac d'Orédon; $65 \%$ de ce charriage s'effectue à la fonte des neiges.

\section{The ecology of a high mountain stream in the Pyrenees}

\section{II. - Chemical conditions}

The chemical features of the river Estaragne and its tributaries were studied in relation to general geographical conditions.

Bicarbonates and calcium formed more than $90 \%$ of the ionic content of the water. The amounts of total iron, $\mathrm{Ca}^{++}, \mathrm{HCO}_{3}^{-}, \mathrm{SO}_{4}^{--}, \mathrm{PO}_{4}-\mathrm{P}, \mathrm{NO}_{3}-\mathrm{N}$, $\mathrm{Mg}^{++}, \mathrm{K}^{+}$decreased from the source of the river to its mouth. Concentrations of $\mathrm{Na}^{+}, \mathrm{Cl}^{-}$and $\mathrm{SiO}$, remained constant or increased slightly down the length of the river. Conductivity changes from $114 \mu$ mhos to $80 \mu$ mhos between the source and the mouth of the river. The changes in the chemical composition of the water are related to the changes in the stream bottom from an unstable and naked bedrock to a crystalline bedrock covered with plants.

Most of the chemical features showed some significant correlations with flow. It has therefore been possible to estimate (with confidence limits) the quantities of material transported down the stream during the two years of this study. The river discharged into Lake Oredon a mean of $470 \mathrm{t} / \mathrm{year}$ for dissolved elements, $65 \%$ of this material is carried down the river when the snow melts.

1. Ce mémoire constitue une partie d'une thèse présentée à l'Université P. Sabatier de Toulouse en vue de l'obtention du Doctorat ès Sciences Naturelles.

1.-2. Laboratoire d'Hydrobiologie, Univ. P. Sabatier, 118, route de Narbonne, 31077 Toulouse Cedex. 
INTRODUC:TION

1. - Programme d'échantillonnage. Eléments analysés. ........ 277

2. - Résultats.

2.1. - Caractéristiques chimiques générales $\ldots \ldots \ldots \ldots \ldots$

2.2. - Evolution des composantes chimiques au cours de la phase déneigée en $1972 \ldots \ldots \ldots \ldots \ldots \ldots \ldots \ldots$

2.3. - Rapport entre les divers éléments du réseau hydrographique. Charge ionique transportée en phase

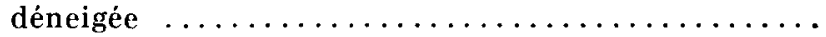

2.4. - Quantités de substances minérales dissoutes déversées annuellement dans le Iac d'Orédon. ..........

3. -... Discussion.

3.1. - Caractéristiques chimiques générales $\ldots \ldots \ldots \ldots \ldots$

3.2. - Evolution des composantes chimiques en fonction du débit .......................... 303

3.3. - Quantités transportées ................ 303

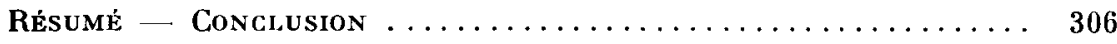

\section{INTRODUCTION}

L'analyse et l'interprétation des caractéristiques chimiques du torrent d'Estaragne et de ses affluents, nécessitent le rappel des quelques données géographiques générales exposées en détail, dans la première partie de l'étude écologique de ce cours d'eau pyrénéen de haute montagne (Lavandier, 1974).

Le vallon d'Estaragne, encaissé dans sa partie haute, est orienté au nord. Les températures y sont froides, la couverture neigeuse importante et prolongée (6-7 mois à $2400 \mathrm{~m} ; 3-5$ mois à $1850 \mathrm{~m}$ d'altitude). Il est drainé par un torrent froid issu de sources dont la température n'excède pas $2,5^{\circ} \mathrm{C}$.

Le bassin versant, d'une altitude moyenne élevée $(+$ de $50 \%$ au-dessus de $2500 \mathrm{~m}$ ), est peu étendu $\left(6,5 \mathrm{~km}^{2}\right)$. Il est formé de schistes, de pélites, de calcschistes et de granites amphiboliques que le torrent traverse successivement de la source $(2380 \mathrm{~m})$ à l'embouchure (lac d'Orédon $1850 \mathrm{~m}$ ).

La végétation est inexistante dans les zones supérieures; elle s'étoffe vers le lac d'Orédon : aux roches nues succèdent une prairie subalpine, puis une rhodopineraie humide dans le bas du vallon.

Le régime hydrique est un régime nival. En été, les orages peuvent provoquer de fortes crues; l'eau de ruissellement, retenue par les éléments de schistes et de pélites désagrégés, maintient alors, durant plusieurs jours, un débit important. 


\section{1. - PROGRAMME D'ÊCHANTILLONNAGE. ELEMENTS ANALYSES.}

Les premiers prélèvements réguliers ont été réalisés en 1970 dès la mise en service d'une station limnigraphique de référence (station R, $2150 \mathrm{~m}$ d'altitude). Durant l'été 1972, les enregistrements de débit furent étendus à tout le réseau hydrographique, et les analyses chimiques effectuées tous les quinze jours à toutes les stations, où le débit pouvait être estimé. Poursuivis jusqu'en 1974, ces relevés sont complétés par l'étude des ruisseaux tempo-

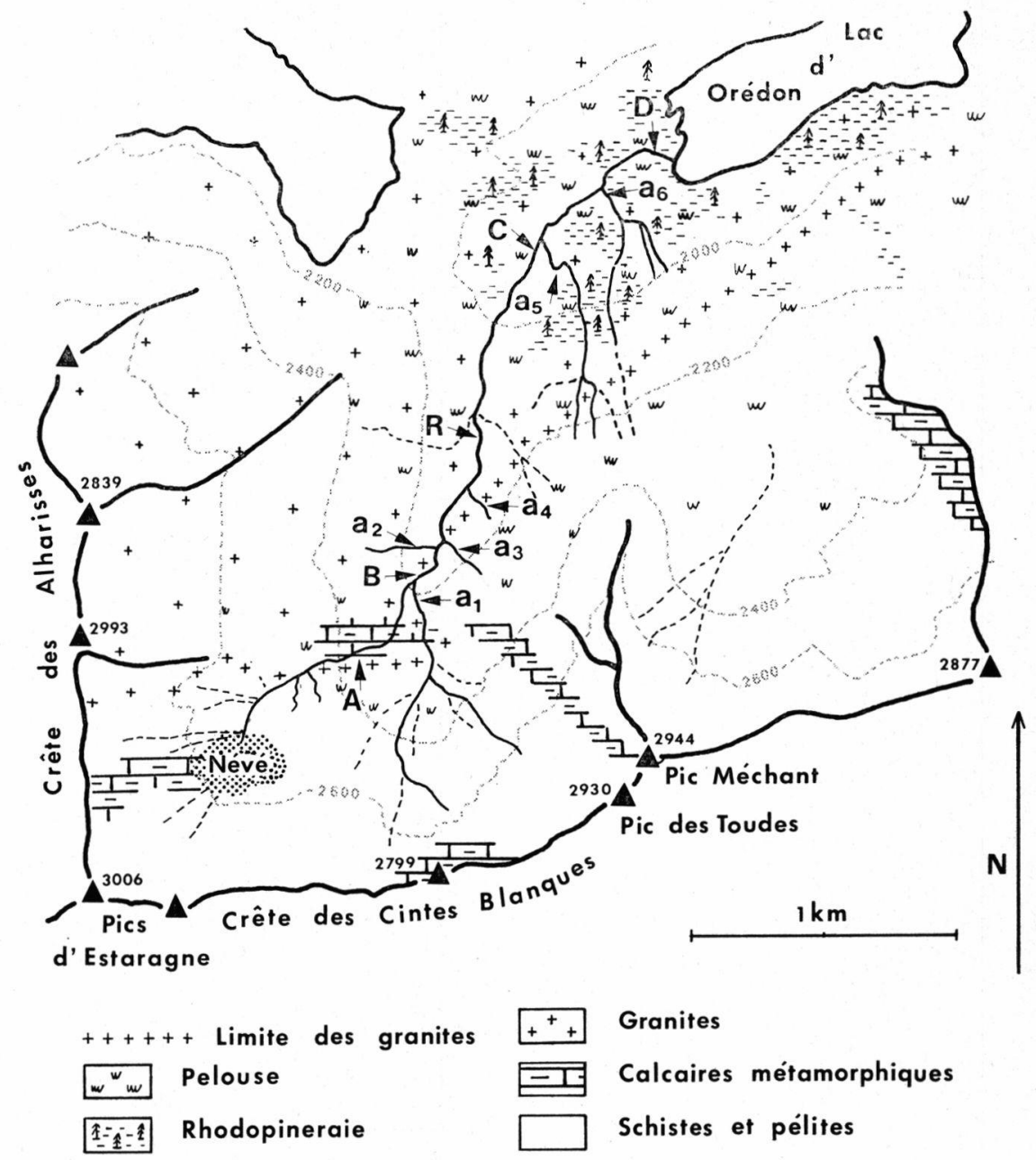

Fig. 1. - Le vallon d'Estaragne. Caractéristiques géographiques générales et stations d'étude. 
raires, des eaux de précipitation et de ruissellement. Sous la couverture de neige hivernale, seuls deux prélèvements ont pu être réalisés (janvier et avril 1970 à la station $R$ ).

La figure 1 situe les localités d'échantillonnage et les grands types de sols du bassin versant.

Les caractéristiques suivantes sont étudiées de façon systématique : conductivité, alcalinité, pH, silice, fer, sulfates, phosphates, nitrates, calcium, magnésium, sodium, potassium; l'oxygène, la dureté totale et les chlorures ont fait l'objet d'analyses moins régulières; le gaz carbonique est calculé. Tous les dosages effectués au laboratoire, excepté celui du fer, sont réalisés sur de l'eau filtrée. Les méthodes utilisées sont décrites dans Standard Methods (1971).

\section{2. - RESULTATS.}

\section{1. - Caractéristiques chimiques générales.}

Elles sont déduites des résultats obtenus en 1972. Leurs valeurs moyennes sont utilisées pour comparer de façon globale, les propriétés chimiques de l'eau aux divers points du vallon.

L'étude détaillée de l'ensemble des données recueillies au cours des quatre années d'observations permet de vérifier la validité des résultats et leur reproductibilité.

\subsection{1. - OXYGÈNE DISSOUS.}

L'oxygène dissous est dosé par la méthode de Winkler sur des échantillons prélevés, durant la journée le long du réseau hydrographique, et lors de cycles de 24 heures à la station $R$. L'oxygène est fixé sur le terrain, puis titré au laboratoire après transport, à l'obscurité, des échantillons.

Toutes les mesures réalisées durant la phase déneigée indiquent des concentrations voisines des valeurs à saturation $(8,5$ à $10,5 \mathrm{mg} / \mathrm{l})$. Seules des différences minimes entre la source et l'embouchure des ruisselets affluents au cirque supérieur, ont pu être décelées : l'eau de source est légèrement moins oxygénée. Par contre, aucune variation altitudinale nette et systématique, aucun cycle nycthéméral n'ont pu être mis en évidence sur le torrent. Même quand se développe une couverture biologique importante, les concentrations en oxygène dissous résultent essentiellement de la diffusion; ce phénomène domine et masque, dans ce milieu torrenticole froid, toute influence biologique.

Les valeurs hivernales sont comparables à celles d'été. 


\subsection{2. - Conductivité ET TENEURS EN SELS DISSOUS.}

La conductivité est mesurée à $20^{\circ} \mathrm{C}$ à l'aide d'un conductimètre Tacussel type CD 6 . Les résultats sont exprimés en $\mu$ mhos.

Divisées par 100 les valeurs de la conductivité donnent une bonne appréciation du contenu ionique (cations ou anions) exprimé en meq./l (Goltermann 1969).

La minéralisation globale est calculée d'après la formule de Doroschewski (Rodier 1966, p. 47).

Tableau I. - Conductivité et teneur en sels dissous : valeurs moyennes aux différentes stations pendant la phase déneigée en 1972 .

\begin{tabular}{|c|c|c|c|c|c|c|c|c|c|c|c|c|}
\hline & ations & A & a1 & $\mathbf{B}$ & a2 & 23 & 24 & $\mathbf{R}$ & $\mathbf{C}$ & a5 & a6 & $\mathbf{D}$ \\
\hline Conductivité & $20^{\circ} \mathrm{C} \quad \mu$ mhos & 114 & 97,5 & 108 & 21,6 & 81,2 & 53,1 & 92,9 & 87,3 & 46,1 & 53,5 & 80,2 \\
\hline Sels dissous & $(\mathrm{mg} / 1)$ & 78,7 & 67,1 & 74,2 & 14,9 & 55,9 & 36,5 & 61,9 & 60,1 & 31,7 & 36,8 & 53,7 \\
\hline
\end{tabular}

Trois groupes de cours d'eau s’individualisent (tableau I) : 1) a2 issu de chaos granitiques est le ruisseau le plus pauvre; sa conductivité très faible n'a jamais dépassé 34 et s'abaisse à 10 lors de la crue nivale ; 2 ) les trois affluents d'aval a4, a5, a6 dominés par des formations de schistes et de pélites ont une conductivité de l'ordre de $50, \mu$ mhos, ce qui correspond à une minéralisation globale de $34,4 \mathrm{mg} / 1$; a1, a3 et l'Estaragne sont influencés par le substrat calcaire qu'ils traversent ou côtoient. C'est à leur niveau que la minéralisation est la plus importante (supéricure à 50). Sur le cours axial, la conductivité, donc la teneur en sels dissous, diminue avec l'altitude pendant que le débit augmente. Il se produit ainsi une dilution des sels, de la source à l'embouchure, au fur et à mesure que le bassin devient granitique. La conductivité moyenne évaluéc en 1972 était de $114 \mu$ mhos à $2350 \mathrm{~m}$, de 92,9 à $2180 \mathrm{~m}$ d'altitude et de 80,2 au niveau du lac. La minéralisation globale passe ainsi de $78,7 \mathrm{mg} / 1$ au cirque supéricur à $53,7 \mathrm{mg} / 1$ à l'embouchure, soit une chute de $25 \%$. Cette baisse de concentration en sels est due à l'apport d'eaux moins minéralisées. Tous les affluents de l'Estaragne, toutes les eaux de ruissellement que nous avons pu doser, présentent en aval de la station $A$, une conductivité plus faible que celle du torrent à leur niveau. Notons que la diminution des concentrations est progressive de la source à l'embouchure, même lorsqu'il n'y a pas d'apport d'eau superficielle, ou de différences de débit mesurables entre deux stations. Ceci suggère la possibilité d'échanges avec un sous-écoulement plus pauvre provenant de terrains cristallins, souvent objets d'une circulation d'eau souterraine intense (Barrère 1952). 


\subsection{3. - Alcalinité, pH, gaz carbonique.}

La neutralisation de l'alcalinité d'un échantillon d'eau par de l'acide sulfurique de titre connu, est réalisée en présence de B.D.H. (mélange de rouge de méthyle et de vert de bromocrésol). Les teneurs sont exprimées en $\mathrm{mg} / \mathrm{l}$ de bicarbonate de calcium - l'ion $\mathrm{HCO}_{3}{ }^{-}$étant dans les conditions de $\mathrm{pH}$ considérées, la forme principale des carbonates (Hutchinson 1957).

Le pH est mesuré sur le terrain, puis au laboratoire à l'aide d'un pH-mètre électrique photovolt. Le $\mathrm{pH}$ à saturation ( $\mathrm{pHs}$ ) est calculé d'après le nomogramme de Hoover-Langelier (in Dussart 1966).

Les quantités de $\mathrm{CO}_{2}$ total sont calculées à partir du $\mathrm{pH}$, de l'alcalinité et de la température, d'après un tableau proposé par Saunders, Trama et Bachmann (1962).

Les tables de Saruhashi (1955) permettent d'évaluer les proportions relatives des différentes formes carbonées (carbonates, bicarbonates, $\mathrm{CO}_{2}$ libre) d'une eau, dont on connaît l'alcalinité, le $\mathrm{pH}$ et la température. Les résultats sont consignés dans le tableau II.

Tableau II, - Alcalinité totale, pH et $\mathrm{CO} 2$ total : valeurs moyennes aux différentes stations pendant la phase déneigée en 1972 .

\begin{tabular}{|c|c|c|c|c|c|c|c|c|c|c|c|}
\hline Stations & A & a1 & B & a2 & a3 & a4 & $\mathbf{R}$ & C & a5 & a6 & D \\
\hline $\begin{array}{l}\text { Alcalinité }\left(\mathrm{mg} / \mathrm{l} \mathrm{HCO}_{\mathrm{a}}^{-}\right) \\
\mathrm{pH} \\
\mathrm{CO}_{2} \text { total }(\mathrm{mg} / \mathrm{l})\end{array}$ & $\begin{array}{l}58,5 \\
8,25 \\
42,2\end{array}$ & $\begin{array}{l}51,6 \\
8,10 \\
38,7\end{array}$ & $\begin{array}{l}55,3 \\
7,97 \\
41,6\end{array}$ & $\begin{array}{r}13,2 \\
7,4 \\
10,7\end{array}$ & $\begin{array}{l}45,7 \\
7,75 \\
35\end{array}$ & $\begin{array}{c}28,9 \\
7,46 \\
23,5\end{array}$ & $\begin{array}{r}49,8 \\
7,8 \\
37,4\end{array}$ & $\begin{array}{c}47,3 \\
7,88 \\
35,5\end{array}$ & $\begin{array}{r}25,6 \\
7,6 \\
19,9\end{array}$ & $\begin{array}{r}30,3 \\
7,7 \\
22,7\end{array}$ & $\begin{array}{l}43,9 \\
7,8 \\
33\end{array}$ \\
\hline
\end{tabular}

L'alcalinité est très faible au niveau des affluents qui coulent essentiellement sur le granite. Les valeurs moyennes sont de 13,2 pour a2, inférieures à 30 pour les ruisseaux d'aval. Sur le torrent, l'alcalinité diminue, comme la conductivité, de l'amont vers l'aval. Elle passe ainsi de $58,5 \mathrm{mg} / 1$ de $\mathrm{HCO}_{3}{ }^{-}$au cirque supérieur à 43,9 à la station $\mathrm{D}$.

Les $\mathrm{pH}$ varient de 8,25 à 7,8 entre la source et l'embouchure du torrent. Les affluents sont un peu moins basiques.

Le $\mathrm{CO}_{2}$ total présente la même évolution que les deux caractéristiques précédentes.

Ces trois paramètres, très liés, soulignent l'importance de la formation des bicarbonates dans les propriétés chimiques des eaux étudiées. Aux pH considérés, l'alcalinité n'est pratiquement due qu'aux bicarbonates dont la formation dépend de la composition cationique de l'eau et de son agressivité.

Dans le cas présent, le calcium représente plus de $90 \%$ des cations et ses concentrations diminuent de l'amont vers l'aval. 
L'agressivité (différence entre $\mathrm{pH}$ et $\mathrm{pHs}$ ) s'élève de la source à l'embouchure de l'Estaragne, alors que le $\mathrm{pH}$ diminue. Le $\mathrm{pH}$, qui dépend du rapport $\mathrm{CO}_{2}$ des bicarbonates $/ \mathrm{CO}_{2}$ libre, s'abaisse, car les concentrations en calcium disponible s'amenuisant, le $\mathrm{CO}_{22}$ des bicarbonates diminue et le $\mathrm{CO}_{12}$ libre (équilibrant et agressif) augmente légèrement.

Le $\mathrm{CO}_{2}$ total est presque entièrement sous forme de bicarbonates ; les pourcentages sont de l'ordre de $90 \%$ sur l'ensemble du réseau - les plus bas sont relevés en a2 (79\%). Les teneurs en $\mathrm{CO}_{2}$ total sont donc étroitement liées à celles de l'alcalinité. La proportion de $\mathrm{CO}_{2}$ libre va de pair avec l'agressivité et les ruisseaux les plus agressifs sont les moins minéralisés.

Le rapport conductivité des carbonates/conductivité totale, déduit des valeurs de Ruttner (1953), permet d'apprécier la proportion en carbonates de l'eau. Ses valeurs moyennes s'établissent entre 74 et $82 \%$. Ces ahiffres sont proches de ceux trouvés par Capblaneq et Laville (1968) pour neuf laes du massif granitique de Néouvielle. Estimées d'après l'abondance relative du $\mathrm{CO}_{2}$ des bicarbonates dans le $\mathrm{CO}_{2}$ total, ou calculées en méq. à partir de l'alcalinité, les proportions sont plus élevées : elles varient de 83 à $92 \%$. Toutefois, quelle que soit la méthode d'évaluation utilisée, ce rapport augmente de la source à l'embouchure du torrent, à l'inverse de la conductivité totale. Par contre, l'alcalinité (donc les bicarbonates) varie dans le même sens que la minéralisation de l'eau. La diminution des teneurs en carbonates est donc moins rapide que celle des concentrations en sels dissous. De fait, les eaux de ruissellement recueillies sur terrain granitique présentent des proportions de carbonates plus fortes que celles du torrent.

\subsection{4. - Sillice.}

En milieu acide ( $\mathrm{pH} 3$ à 4) la silice forme avec le molybdate d'ammonium un complexe jaune : la réduction de ce complexe par un acide aminé (acide amino-naphtol-sulfonique) donne une coloration bleue dont l'intensité est mesurée au spectrophotomètre à $800 \mathrm{~nm}$.

Les concentrations en silice (tableau III) sont faibles, inféricures à $4 \mathrm{mg} / \mathrm{l}$. C'est le cours d'eau a1, issu des schistes et des pélites sud-est, qui est le plus pauvre ; il détermine après sa confluence, une chute de la teneur en $\mathrm{SiO}_{2}$ sur le torrent. Par la suite, la concentration moyenne s'élève de l'amont vers l'aval ; cette évolution est d'autant plus nette que les débits sont faibles. Durant la fonte des neiges, les analyses révèlent une certaine homogénéisation des teneurs à tous les niveaux. Les affluents ont alors des 
concentrations légèrement supérieures à celles du torrent, mais l'eau de ruissellement est plus pauvre. A l'étiage, tous les affluents sont plus riches en silice que le cours axial.

La silice n'entre que pour une faible part dans la minéralisation totale. Elle représente $3 \%$ des sels dissous au cirque supérieur et $5 \%$ à la station $\mathrm{D}$.

TABleau III. - Silice et fer total : valeurs moyennes aux différentes stations pendant la phase déneigée en 1972.

\begin{tabular}{lccccccccccc}
\hline Stations & A & a1 & B & a2 & a3 & a4 & R & C & a5 & a6 & D \\
\hline $\mathrm{SiO}_{2}(\mathrm{mg} / \mathrm{l})$ & 2,61 & 2,07 & 2,54 & 2,58 & 2,76 & 3,28 & 2,62 & 2,80 & 3,76 & 2,93 & 2,84 \\
Fer total $(\mu \mathrm{g} / \mathrm{l})$ & 137 & & & 7 & & 32 & 31,7 & 25 & 29,3 & 86,6 & 15,1 \\
\hline
\end{tabular}

\subsection{5. - FER TOTAL.}

L'orthophénanthroline est utilisée comme réactif. Le fer total est libéré sous forme ionique en milieu chlorhydrique à ébullition, puis réduit par du chlorhydrate d'hydroxylamine. Les ions $\mathrm{Fe}^{++}$ donnent avec l'orthophénanthroline un complexe rouge qui fait l'objet d'un dosage colorimétrique.

Les teneurs observées (tableau III) sont relativement élevées au cirque supérieur où elles atteignent $300 \mu \mathrm{g} / 1$ en période de crue. Elles s'abaissent régulièrement de l'amont vers l'aval.

\subsection{6. - Chlorures.}

Ils sont dosés par la méthode colorimétrique au nitrate mercurique, en présence d'un indicateur coloré : la diphényl-carbazone. La solution de nitrate mercurique, de titre connu, est versée jusqu'à l'obtention d'une couleur violet-clair, indicatrice du virage.

Les concentrations relevées (tableau IV) sont de l'ordre de 0,5 à $0,75 \mathrm{mg} / \mathrm{l}$ sur l'ensemble du réseau et ne semblent guère varier au cours de la saison. En fait, la méthode utilisée ne permet pas de déterminer plus précisément l'évolution altitudinale ou saisonnière de teneurs aussi faibles.

Tableau IV. - Chlorures et sulfates : valeurs moyennes aux différentes stations pendant la phase déneigée en 1972.

\begin{tabular}{llllllllllll}
\hline \multicolumn{1}{c}{ Stations } & A & al & B & a2 & a3 & a4 & R & C & a5 & a6 & D \\
\hline $\mathrm{CI}^{-}(\mathrm{mg} / 1)$ & 0,5 & 0,75 & $\mathbf{0 , 5}$ & & 0,75 & 0,5 & $\mathbf{0 , 7 5}$ & 0,75 & 0,75 & 0,75 & 0,75 \\
$\mathrm{SO}_{4}=(\mathrm{mg} / \mathrm{l})$ & 6,44 & $\mathbf{3 , 5}$ & $\mathbf{5 , 3 7}$ & 2 & 3,25 & $\mathbf{2 , 4 4}$ & 4,78 & $\mathbf{4 , 2 7}$ & $\mathbf{2 , 2 7}$ & $\mathbf{2 , 2}$ & $\mathbf{3 , 6 1}$ \\
\hline
\end{tabular}




\subsection{7. - Sulfates.}

L'ion $\mathrm{SO}_{4}^{--}$est évalué par turbidimétrie. En milieu chlorhydrique, les sulfates sont précipités à l'état de sulfates de baryum par addition de chlorure de baryum stabilisé. Les déviations lues sont traduites en concentrations, par référence à une courbe d'étalonnage pré-établie.

Les teneurs sont maximales dans la partie haute du vallon, $6,44 \mathrm{mg} / \mathrm{l}$ à la station A (tableau IV). Elles diminuent vers l'aval, les affluents étant tous moins riches que le cours principal à altitude équivalente. Les concentrations les plus faibles sont enregistrées sur a2 mais constituent dans ce cours d'eau peu minéralisé $15 \%$ des sels dissous. Sur le torrent, ces proportions sont voisines de $10 \%$; elles s'abaissent de la source à l'embouchure : la dilution des sulfates est donc plus rapide que celle des sels pris dans leur ensemble. L'évolution des concentrations avec l'altitude n'est pas constante au cours de la saison : la dilution est forte $(50 \%)$ quand les débits sont faibles, elle s'annule lors des crues. $\mathrm{SO}_{4}^{--}$est en grande partie responsable de la dureté permanente dont les valeurs ont montré, lors des quelques dosages réalisés, une évolution semblable à celle de cet anion.

\subsection{8. - Phosphates.}

En milieu acide le molybdate d'ammonium forme avec les orthophosphates un complexe phosphomolybdique. Sa réduction par le chlorure stanneux (Strickland et Parsons 1960) libère une coloration bleue dont l'intensité est mesurée au spectrophotomètre à $690 \mathrm{~nm}$. L'extraction du complexe phosphomolybdique, réduit par un solvant organique, l'isobutanol, rend la méthode plus sensible et permet le dosage de teneurs de l'ordre de quelques microgrammes.

Les phosphates présentent en Estaragne des teneurs moyennes inférieures à $5 \mu \mathrm{g} / \mathrm{l}$ (tableau V). Malgré ces concentrations extrêmement faibles, une dilution de l'amont vers l'aval apparaît nettement dans le torrent principal. L'évolution irrégulière, que présentent souvent les valeurs entre deux stations successives, doit être rattachée au développement inégal de la végétation en cours de saison.

Tableau V. - Orthophosphates et nitrates : valeurs moyennes aux différentes stations pendant la phase déneigée en 1972.

\begin{tabular}{lcrrrrrrrrrrr}
\hline Stations & A & a1 & B & a2 & a3 & a4 & R & C & a5 & a6 & D \\
\hline $\mathrm{PO}_{4}-\mathrm{P}(\mu \mathrm{g} / 1)$ & 4,84 & 2,3 & 1,86 & 1,7 & 3,2 & 1,57 & 1,88 & 2,09 & 1,5 & 1,9 & 2,01 \\
$\mathrm{NO}_{3}-\mathrm{N}(\mu \mathrm{g} / 1)$ & 192 & 186 & 155 & 141 & 149 & 218 & 144 & 142 & 90,5 & 179 & 160 \\
\hline
\end{tabular}




\subsection{9. - Nitrates.}

L'échantillon à analyser est évaporé à sec, au bain-marie, en présence de salicylate de sodium. Le résidu est dissous dans $\mathrm{H}_{2} \mathrm{SO}_{4}$ concentré. Après addition d'eau distillée, la solution est alcalinisée par $\mathrm{NaOH}$. Le dérivé obtenu prend une coloration jaune estimée au spectrophotomètre à $420 \mathrm{~nm}$, après refroidissement. Les résultats sont exprimés en $\mathrm{mg} / \mathrm{l}$ de $\mathrm{NO}_{3}-\mathrm{N}$ (tableau $\mathrm{V}$ ).

Les concentrations sont faibles et dépassent rarement $0,2 \mathrm{mg} / 1$. Les affluents a4 et a1, puis l'Estaragne au cirque supérieur, sont les plus riches $(180-220 \mu \mathrm{g} / \mathrm{l})$. Sur le torrent, les teneurs moyennes diminuent de l'amont jusqu'à la station $C$, pour s'élever ensuite légèrement au niveau du lac d'Orédon. La diminution des nitrates est maximum à l'étiage. Ces ions constituent moins de $0,05 \%$ du total des sels dissous. Les variations de la teneur en nitrates ne présentent pas de corrélation nette avec celles de la minéralisation globale.

\subsubsection{0. - Calcium.}

Les teneurs en $\mathrm{Ca}^{++}$(tableau VI) exprimées en $\mathrm{mg} / \mathrm{l}$ sont déduites des valeurs de la dureté calcique. A $\mathrm{pH}$ élevé, 12-13, le complexon se combine avec le calcium, et le magnésium est précipité. Un produit HACH (Cal ver II), dont le virage du rouge au bleu est très net, est utilisé comme indicateur.

TABLeaU VI. - Calcium et magnésium : valeurs moyennes aux différentes stations pendant la phase déneigée en 1972.

\begin{tabular}{|c|c|c|c|c|c|c|c|c|c|c|c|}
\hline Stations & A & al & B & a2 & a3 & a5 & a6 & $\mathrm{D}$ & $\mathbf{a} 4$ & $\mathbf{R}$ & $\mathrm{C}$ \\
\hline $\mathrm{Ca}^{++}(\mathrm{mg} / \mathrm{l})$ & 22,6 & 19,2 & 21,2 & 4,5 & 15 & 9,7 & 17,6 & 17 & 9 & 10 & 15,6 \\
\hline $\mathrm{Mg}^{++}(\mathrm{mg} / \mathbf{l})$ & 0,392 & 0,205 & 0,295 & 0,147 & 0,263 & 0,362 & 0,284 & 0,286 & 0,296 & 0,239 & 0,322 \\
\hline Rapport Mg/Ca & 0,017 & 0,011 & 0,014 & 0,033 & 0,017 & 0,037 & 0,016 & 0,017 & 0,033 & 0,024 & 0,021 \\
\hline
\end{tabular}

Le calcium représente généralement plus de $90 \%$ des cations à toutes les stations, les concentrations les plus fortes sont liées à la présence de roches calcaires. Sur le torrent la diminution des concentrations est d'autant plus sensible que l'on s'éloigne du cirque supérieur $(22,6 \mathrm{mg}$ à la station $\mathrm{A} ; 15,6$ à la station $\mathrm{D})$. L'affluent a2 provenant de rochers granitiques, est le plus pauvre $(4,5 \mathrm{mg} / \mathrm{l})$; les cours d'eau qui traversent les schistes présentent des teneurs intermédiaires $(9-19 \mathrm{mg} / \mathrm{l})$.

Les proportions de $\mathrm{Ca}^{++}$par rapport aux sels dissous diminuent légèrement $(2 \%)$ de la source à l'embouchure du cours axial. Les concentrations en $\mathrm{Ca}^{++}$sont évidemment étroitement liées à la 
minéralisation totale dont elles constituent une part importante. Exprimées en milliéquivalent, les tencurs en $\mathrm{Ca}^{++}$sont égales ou supérieures à celles des bicarbonates; en $\mathrm{mg} / \mathrm{l}$ elles en représentent en moyenne $35 \%$.

\section{2:1.11. - $\mathrm{Mg}^{++}, \mathrm{Na}^{+}, \mathrm{K}^{+}$}

Ces cations sont dosés directement par spectrophotométrie d'absorption atomique (spectrophotomètre Pye Unicam SP 90). Le magnésium est déterminé en absorption ; le sodium et le potassium en émission.

Tableau VII. - Sodium et potassium : valeurs moyennes aux différentes stations pendant la phase déneigée en 1972 .

\begin{tabular}{|c|c|c|c|c|c|c|c|c|c|c|c|}
\hline Stations & $A$ & a1 & B & a2 & a3 & $\mathbf{a} 4$ & $\mathrm{R}$ & C & a5 & a6 & D \\
\hline $\mathrm{Na}^{+}(\mu \mathrm{g} / 1)$ & 501 & 511 & 481 & 486 & 559 & 480 & 444 & 488 & 654 & 528 & 504 \\
\hline $\mathrm{K}^{+}(\mu \mathrm{g} / \mathrm{l})$ & 335 & 271 & 358 & 211 & 330 & 336 & 266 & 283 & 210 & 312 & 306 \\
\hline Rapport $\mathrm{K} / \mathrm{Na}$ & 0,668 & 0,530 & 0,744 & 0,434 & 0,592 & 0,700 & 0,599 & 0,579 & 0,321 & 0,590 & 0,600 \\
\hline
\end{tabular}

Ces trois éléments ne sont présents qu'en faible quantité (tableaux VI et VII) et constituent moins de $10 \% \mathrm{du}$ total des cations. Les teneurs moyennes maximales sont : $392 \mu \mathrm{g} / 1 \mathrm{de} \mathrm{Mg}^{++}$ (station A), $654 \mu \mathrm{g} / \mathrm{l}$ de $\mathrm{Na}^{+}$(station a5), $358 \mu \mathrm{g} / \mathrm{l} \mathrm{de} \mathrm{K}^{+}$(station B). D'une manière générale, les teneurs sont assez homogènes sur l'ensemble du réseau étudié.

Sur le torrent, on note une diminution des concentrations en $\mathrm{Mg}^{++}$el $\mathrm{K}^{+}$vers l'aval, suivie d'une légère augmentation dans le cours inférieur ; $\mathrm{Na}^{+}$reste en quantité relativement constante. Leur importance dans la minéralisation globale s'accroît de la source à l'embouchure. La dilution de ces cations est donc proportionnellement plus faible que celle des autres ions, sur l'ensemble du cours. Le rapport $\mathrm{Mg} / \mathrm{Ca}$ en $\mathrm{mg} / \mathrm{l}$ est très faible, de l'ordre de 0,02 . Il est plus élevé dans les stations les plus pauvres en calcium. Le rapport $\mathrm{K} / \mathrm{Na}$ est généralement compris entre 0,5 et 0,75 .

\subsubsection{2. - Remarque.}

Au cirque supérieur l'écoulement superficiel n'apparaît qu'une soixantaine de mètres en amont de la station $A(2370 \mathrm{~m}$ d'altitude). A cette station toutefois, ses caractéristiques générales ne sont pas comparables à celles d'une eau de source typique. En effet, l'eau a déjà plusieurs origines. Elle provient : 1) de la fonte progressive du névé ; 2 ) des cascades qui descendent de la crête des Cintes Blanques, puis s'écoulent sous les éboulis granitiques 
avant de former deux petites sources limnocrènes ; 3) de ruisseaux et rigoles qui traversent des tourbières bombées à Hypnacées ; 4) de ruissellements sous-jacents. C'est l'importance relative de ces différents apports qui détermine la composition chimique observée à la station A.

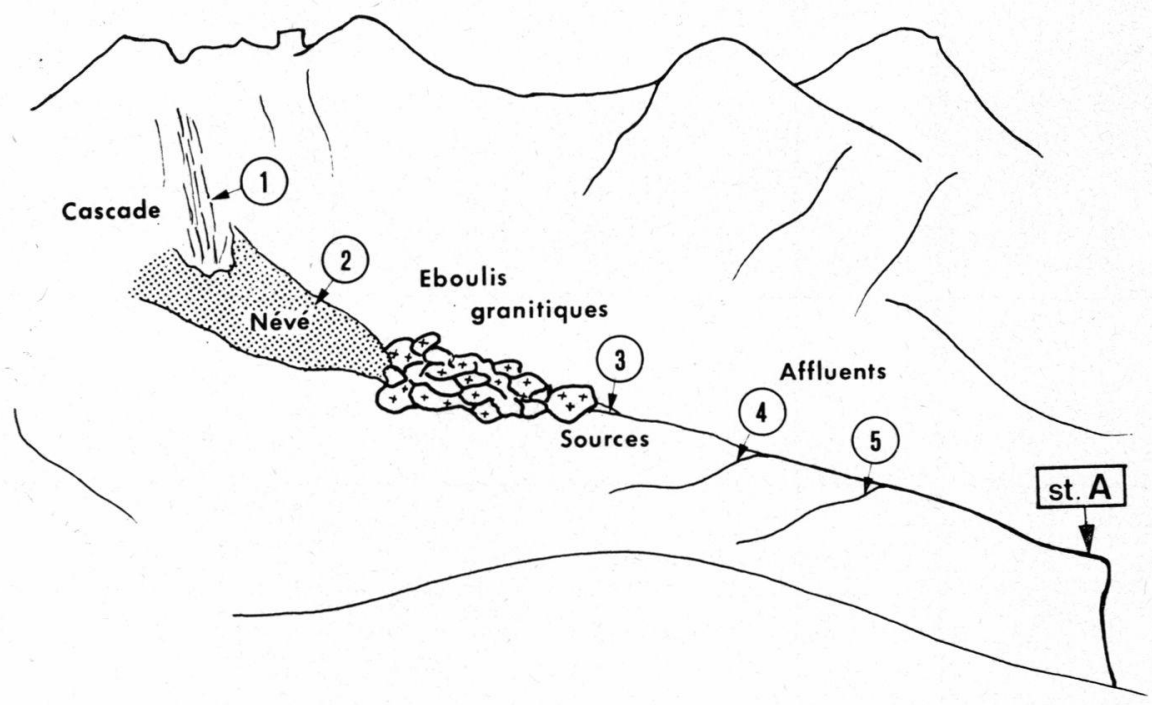

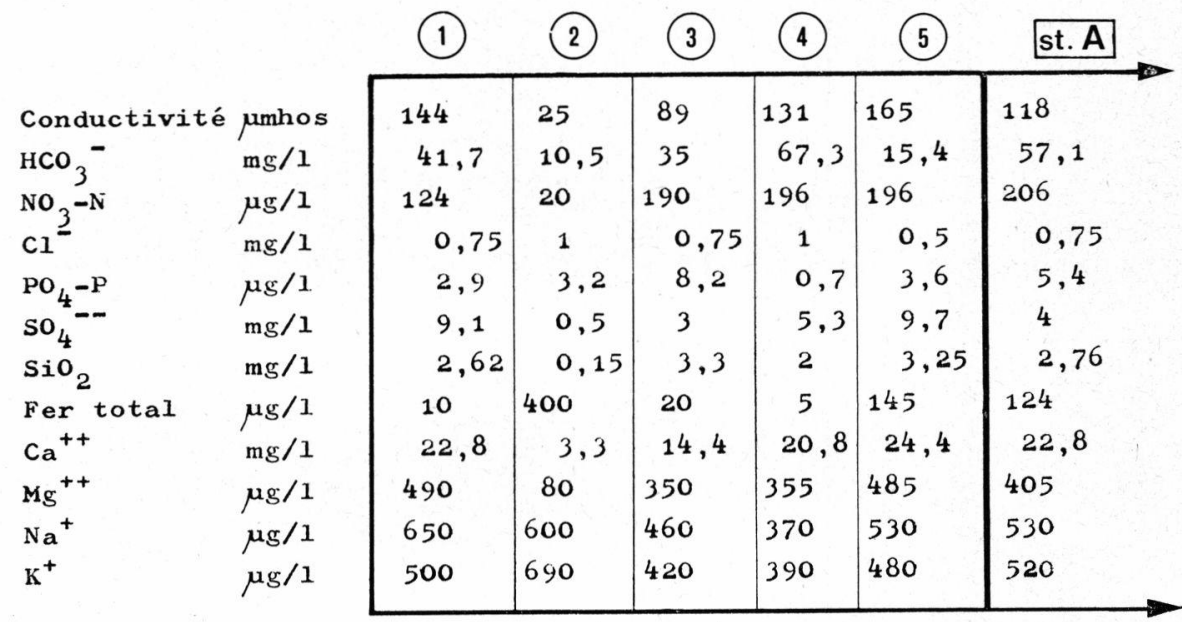

Fig. 2. - Origines de la composition chimique de l'eau à la station A.

La figure 2 montre l'acquisition progressive des caractéristiques chimiques de l'eau à cette station au début du mois de septem- 
bre 1973 (débit voisin de $100 \mathrm{l} / \mathrm{s}$ ). Les modifications chimiques observées ici sont nettes, étant donné l'origine différente des eaux d'alimentation. Elles mettent en évidence, en quelques mètres et de façon tranchée, les phénomènes plus nuancés, plus progressifs, qui se produisent tout au long du cours axial.

\section{2. - Evvolution des composantes chimiques au cours de la phase déneigée en 1972.}

Cette évolution traduit en fait les relations existant entre les propriétés chimiques de l'eau et le débit. Après transformation logarithmique des valeurs de l'écoulement, nous avons calculé les équations de corrélation - et les limites de confiance au seuil $\mathbf{P}=0,05$ - pour chaque caractéristique à toutes les stations. La figure 3 présente les relations établies aux stations situées sur le cours axial, objet principal de notre étude.

- Deux types de composantes chimiques apparaissent :

* conductivité, silice, alcalinité, $\mathrm{Ca}^{++}, \mathrm{Na}^{+}, \mathrm{Mg}^{++}, \mathrm{SO}_{4}^{--}$et $\mathrm{NO}_{3}-\mathrm{N}$ à la station $\mathrm{A}$ présentent une corrélation négative avec les débits ;

* $\mathrm{NO}_{3}-\mathrm{N}$ et $\mathrm{SO}_{4}^{--}$(aux stations $\mathrm{B}, \mathrm{R}, \mathrm{C}$ el D), le fer total (aux stations $\mathrm{R}, \mathrm{C}$ et $\mathrm{D}), \mathrm{K}^{+}$(station $\mathrm{B}$ ) et $\mathrm{PO}_{4}-\mathrm{P}$ sont corrélés positivement.

Une indication de corrélation positive mais non significative apparaît pour $\mathrm{K}^{+}$(aux stations $\mathrm{A}, \mathrm{R}, \mathrm{C}, \mathrm{D}$ ), $\mathrm{SO}_{4}^{--}$(à la station $\mathrm{R}$ ) et pour le fer total (station A). Les quelques analyses de $\mathrm{Cl}^{-}$ indiquent pour cet ion une tendance à diminuer quand les débits s'élèvent.

Les pentes des droites, obtenues pour chaque élément aux diverses stations, ont été comparées deux à deux. Elles ne diffèrent significativement qu'entre le cirque supérieur (station A) et l'ensemble des stations d'aval, et pour trois éléments seulement: nitrates, calcium, sulfates. Ce résultat reflète l'homogénéité du bassin versant vis-à-vis des autres composantes, mais aussi l'influence des zones supérieures sur la composition chimique de l'eau dans le réseau hydrographique. Cette influence s'amenuise au fur et à mesure que l'on s'éloigne de la source : les valeurs du coefficient t de Student, utilisé pour comparer les pentes des équations de corrélation «concentration en $\mathrm{Ca}^{++}$- débit », sont à cet égard particulièrement significatives (tableau VIII). 

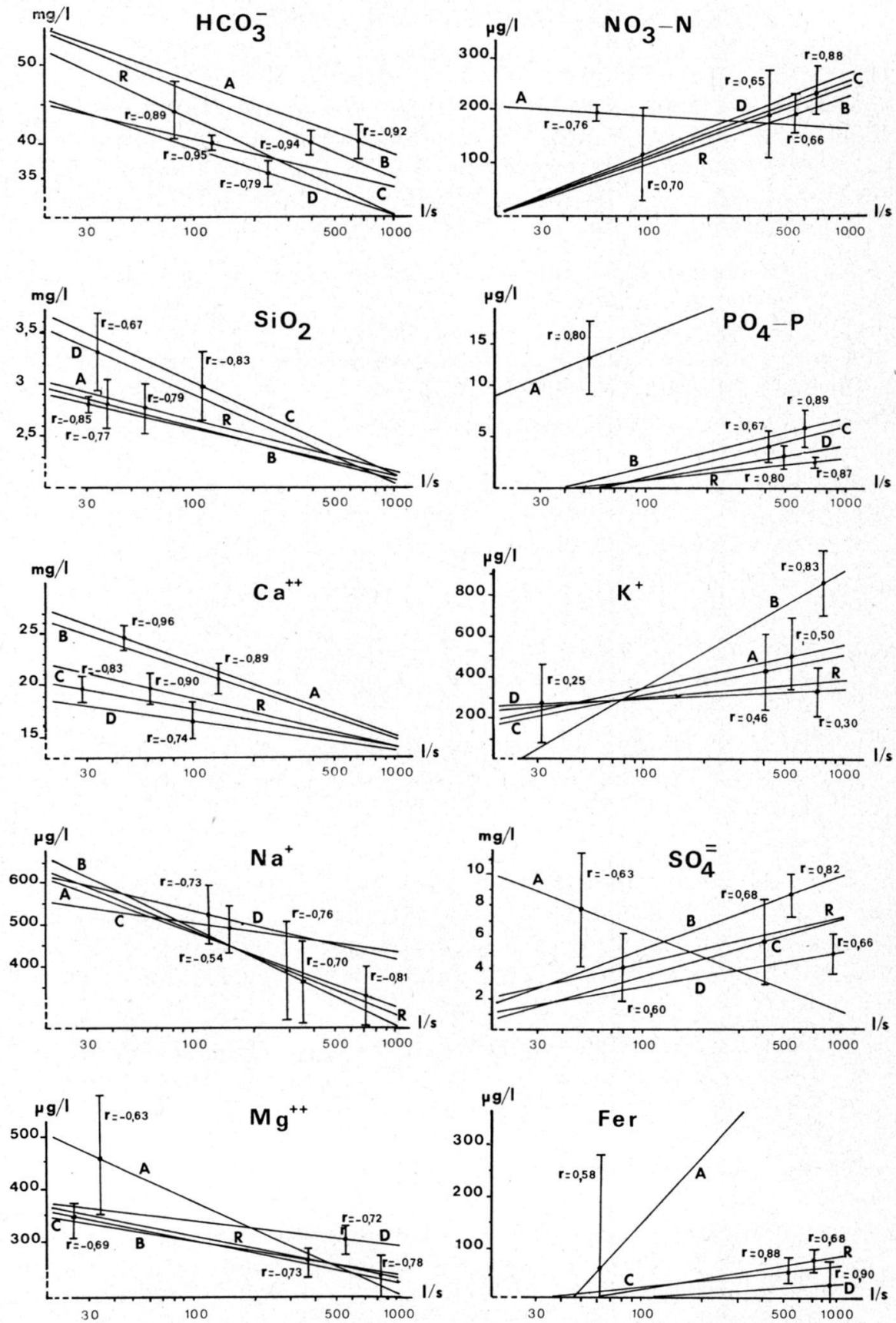

Fig. 3. - Relations entre les composantes chimiques et les débits aux stations $\mathrm{A}, \mathrm{B}, \mathrm{R}, \mathrm{C}$ et $\mathrm{D}$ du torrent d'Estaragne. Pour chaque droite de corrélation un trait vertical donne l'amplitude de l'intervalle de confiance au seuil $\mathrm{P}=0,05$. Les corrélations sont établies à partir de 10 couples de données à chaque station (D.L. $=8$ ). 
Tabieau VIII. - Comparaison deux à deux des pentes des droites de corrélation "concentration en $\mathrm{Ca}^{++}$— débit» obtenues aux 5 stations du cours principal. Les valeurs du coefficient utilisé (coefficient $t$ de Student) diminuent au fur et à mesure que la distance séparant deux stations augmente.

* Valeurs significatives au seuil $P=0,05$.

\begin{tabular}{ccccc}
\hline Stations & B & R & \multicolumn{1}{c}{ C } & D \\
\hline A & 0,36 & $2,67^{\star}$ & $3,21^{\star}$ & $3,30^{*}$ \\
B & & 1,41 & 2 & 2,08 \\
R & & & 1,28 & 1,9 \\
C & & & & 0,41 \\
\hline
\end{tabular}

-- Ces corrélations établies à partir de données recueillies lors de la fonte des neiges, de crues orageuses et de basses eaux d'été, semblent également applicables aux conditions hivernales. Les analyses des deux prélèvements que nous avons pu effectuer sous la neige, ont donné pour tous les éléments étudiés $\left(\mathrm{Ca}^{++}, \mathrm{PO}_{4}-\mathrm{P}\right.$, alcalinité, $\mathrm{SO}_{4}{ }^{--}$, silice, conductivité) des valeurs qui se situent parfois à la limite supérieure (cas des orthophosphates), mais toujours dans l'intervalle de confiance de l'estimation calculée pour un débit analogue, à partir des données estivales.

- Ces corrélations paraissent reproductibles d'une année sur l'autre. A la station de référence $R$, un nombre suffisant d'analyses a permis de calculer les équations de régression correspondant aux années 1970, 1972, 1973 et 1974, puis de comparer les ordonnées à l'origine et les pentes. Ces dernières ne diffèrent pas significativement : la figure 4 montre à titre d'exemple les corrélations existant chaque année entre la conductivité (mesure globale de la teneur en sels dissous) et le débit.

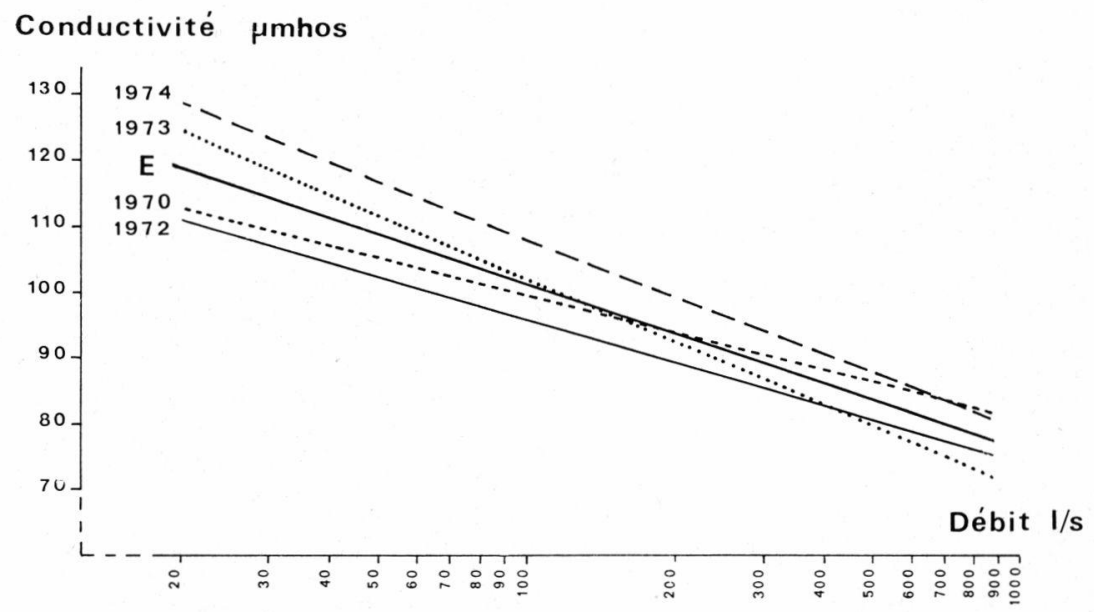

FIG. 4. - Relations entre la conductivité et les débits à la station $\mathrm{R}$ en 1970 , 1972,1973 et 1974. La droite $\mathrm{E}$ intègre l'ensemble des données. 


\section{3. - Rapport entre les divers éléments du réseau hydrographique. Charge ionique transportée en phase déneigée.}

La quantité de matières dissoutes transportées est donnée à chaque instant par le produit concentration - débit. Pour être exploitable, le résultat doit tenir compte des limites de confiance des estimations. Le calcul étant basé sur l'utilisation de corrélations, nous assimilerons les deux droites qui déterminent l'intervalle de confiance (au seuil $\mathrm{P}=\mathbf{0 , 0 5}$ ) d'une corrélation, aux valeurs limites inférieures et supérieures susceptibles d'être rencontrées.

Le problème présent requiert l'emploi successif des relations « hauteur d'eau - débit» et « débit - concentration ». L'intervalle de confiance doit donc être précisé pour l'estimation des débits et des concentrations. L'organigramme de la figure 5 permet d'obtenir les valeurs moyennes (les plus probables), maximales et minimales des débits et des concentrations et par suite de la charge transportée.

La figure 6 résume les conditions chimiques moyennes des eaux en phase déneigée et les relations existant, à cette époque, entre les divers éléments du réseau hydrographique.

- Les quantités transportées augmentent de l'amont vers l'aval; l'augmentation du débit compense donc la dilution généralement observée.

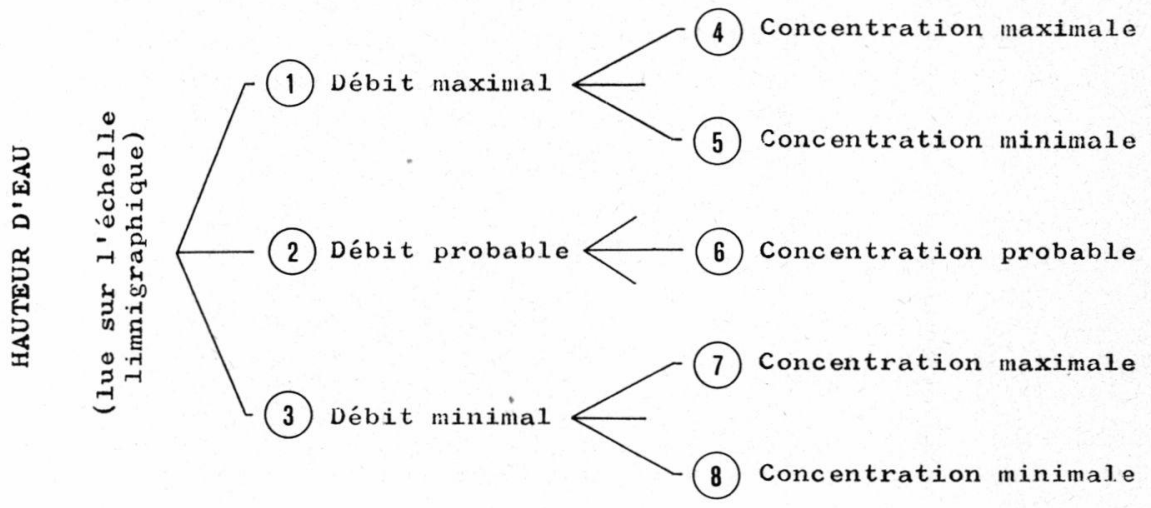

Fig. 5. - Organigramme donnant le mode d'obtention des concentrations et des quantités transportées probables, maximales et minimales envisageables à partir de la hauteur d'eau enregistrée.

- Corrélations négatives : les concentrations extrêmes envisageables sont données en 7 (concentration maximale) et en 5 (concentration minimale).

- Corrélations positives : les concentrations extrêmes envisageables sont données en 4 (concentration maximale) et en 8 (concentration minimale). Dans les deux cas les produits : débit 1-concentration 4 et débit 3-concentration 8 donnent les valeurs maximales et minimales envisageables des quantités transportées.

- Les concentrations les plus probables sont données en 6; les quantités transportées les plus probables sont données par le produit : concentration 6débit 2 . 
- Les quantités globales transportées, calculées à chaque station lors de la période déneigée donnent une image générale moyenne de l'évolution de la charge en fonction de l'altitude. Ce transport varie en fait selon les modalités d'alimentation : * à la fin de la fonte des neiges, plus de la moitié de l'écoulement évalué à la station $D$, provient du cirque supérieur; les quantités de sels dissous sont en moyenne multipliées par 1,5 entre les deux stations extrêmes;

* lors des forts débits d'orage, l'eau issue du cirque supérieur constitue au niveau du lac environ $45 \%$ du débit, essenticllement dû alors au ruissellement; le transport est muliplié par 2,3 ;

* à l'étiage, le débit à la station $D$ résulte à parts égales de l'eau provenant du cirque d'amont et du reste du bassin versant; le transport est multiplié par 1,7, coefficient intermédiaire aux deux précédents.

Ces différences étaient déjà prévisibles lors de l'établissement des corrélations générales : la limite supérieure des estimations est en effet essentiellement déterminée par les concentrations observées après orage, la limite inférieure par celles trouvées à la fonte des neiges.

- Selon les éléments considérés, les quantités transportées évoluent différemment de l'amont vers l'aval; la charge en calcium est multipliée par 1,6 , celle en sodium par 2,5 entre la source et l'embouchure.

- Les affluents amènent environ $40 \%$ des substances déversées dans le lac ( $30 \%$ sont dus à l'affluent a1).

- L'influence des zones supérieures, fondamentale dans l'alimentation du système, est encore plus marquée en ce qui concerne les substances dissoutes. Le transport est multiplié par 2 entre la source et l'embouchure alors que les débits le sont par 2,3. A la station B (après la confluence avec a1 - $2200 \mathrm{~m}$ d'altitude) l'Estaragne porte déjà $70 \%$ des sels dissous qu'il amènera au lac. Les quantilés charriées par $\mathrm{km}^{2}$ de bassin versant sont quatre fois et demi plus fortes à $2370 \mathrm{~m}$ qu'à $1850 \mathrm{~m}$ d'altitude.

\section{4. - Quantités de substances minérales dissoutes déversées annuellement dans le lac d'Orédon.}

Ce calcul nécessite l'évaluation de l'écoulement total annuel au niveau du lac. Il est déterminé par référence aux débits enregistrés à la station $R$, seule station susceptible de fonctionner en hiver. Les enregistrements effectués à tous les niveaux durant la phase déneigéc en 1972 nous permettent d'établir la corrélation et ses limites de confiance - existant entre les débits mesurés aux stations $\mathrm{R}$ et $\mathrm{D}$ (fig. 7 ). Cette relation qui sous-entend une évolution 
parallèle des débits aux deux stations, entraîne une sous-évaluation importante du volume d'eau apporté au lac lors de la fonte des neiges. En effet, le déneigement (donc la crue nivale) commence à $1850 \mathrm{~m}$ d'altitude un mois plus tôt qu'à $2150 \mathrm{~m}$. Une correction s'avère nécessaire. L'ajustement proposé dans la figure 8 , suppose que l'augmentation des débits au niveau du lac, présente les mêmes caractéristiques qu'à la station $R$, le phénomène étant simplement décalé dans le temps.

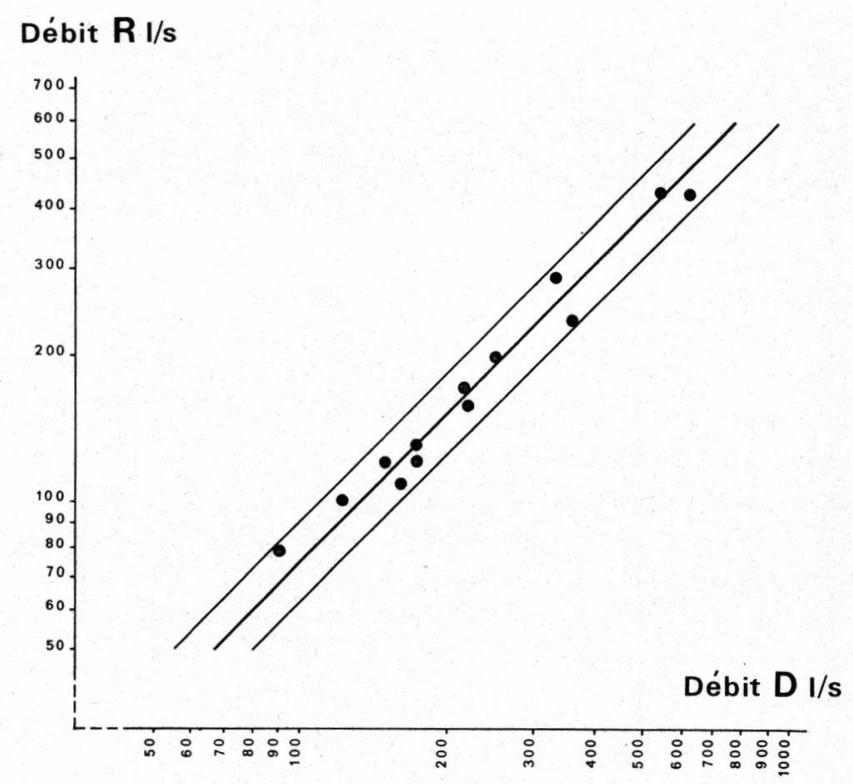

Fig. 7. - Relation entre les débits enregistrés à la station $R$ et les débits enregistrés à la station $\mathrm{D}$. La droite de corrélation et les limites de confiance au seuil $\mathrm{P}=0,05$ sont établies à partir des débits moyens hedbomadaires, calculés aux deux stations pendant la période déneigée du 14-7 au 11-11-1972.

Les quantités transportées sont calculées pour les années hydrologiques 1969-70 et 1970-71, périodes d'enregistrement continu à la station R. Ces estimations se font suivant l'organigramme de la fig. 5, complété seulement par l'introduction de la nouvelle corrélation (débit en R, débit en $\mathrm{D}$ ) — et de ses limites de confiance en début de calcul.

La figure 8 montre l'évolution du transport en cours d'année et indique les charges globales amenées au lac. Elle met en évidence l'importance fondamentale de l'écoulement et la régularité des décharges annuelles.

- La fonte des neiges dure très longtemps et progresse de l'aval vers l'amont; les quantités transportées pendant cette période 
représentent en moyenne plus de $65 \%$ de la charge annuelle. La faible minéralisation de l'eau est largement compensée par l'énorme volume d'eau charriée.

- Sous la neige hivernale, le transport est pratiquement négligeable.

- Pendant la phase déneigée, il varie selon l'écoulement mais n'atteint jamais des valeurs élevées. Ces valeurs peuvent toutefois être sous-estimées à l'entrée de l'hiver : les précipitations, pluvieuses en bas, sont alors neigeuses en altitude. L'évolution des débits en $D$ ne suit pas, alors, celle enregistrée en $R$.

Les volumes d'eau écoulée étant peu différents $\left(4,2 \mathrm{Mm}^{3}\right.$ contro $4,9 \mathrm{Mm}^{3}$ ), les deux années présentent des changes globales transportées voisines : 451 tonnes en 1969-70, 497 tonnes en 1970-71. Ces valeurs intègrent les quantités de chlorures estimées à 2,1 tonnes pour la première année et à 2,5 tonnes pour la seconde. L'écart enregistré entre les deux décharges annuelles est inférieur à $10 \%$. Il est plus faible pour les composantes qui présentent une corrélation positive avec les débits (parmi lesquels se trouvent les éléments dominants $\mathrm{Ca}^{++}, \mathrm{HCO}_{3}^{-}$et $\mathrm{SiO}_{2}$ ) ; il est plus fort pour les autres.

\section{3. - DISCUSSION}

\section{1. - Caractéristiques chimiques générales.}

\subsection{1. - MÉTHOdE EMPLOYÉE.}

Nous avons considéré ci-dessus que les moyennes des valeurs obtenues par dosage étaient représentatives des caractéristiques chimiques générales du torrent. Or ces valeurs varient en fonction du débit. Cette démarche pose donc le problème du nombre de prélèvements et de leur répartition dans le temps. Pour avoir un sens, toute moyenne doit résulter d'un échantillonnage qui reflète le plus exactement possible l'évolution du milieu. Dans le cas présent, le nombre de prélèvements doit être proportionnel à la duréc des différentes phases hydrologiques. Bien que ne réalisant pas pleinement ces conditions, les prélèvements effectués en 1972 donnent une bonne image des propriétés chimiques en phase déneigée. Il y a en effet peu de différences entre les valeurs probables théoriques, qui intègrent toutes les conditions d'écoulement, et les valeurs moyennes déduites directement du résultat des analyses (comparer fig. 6 et tableaux II à VII). Dans le détail nos valeurs sont néanmoins influencées par les conditions chimiques régnant lors des basses eaux. Ainsi, par rapport aux moyennes théoriques, nos valeurs sont légèrement plus fortes pour les éléments qui présentent une corrélation négative avec les débits, légèrement plus faibles dans le cas contraire. 


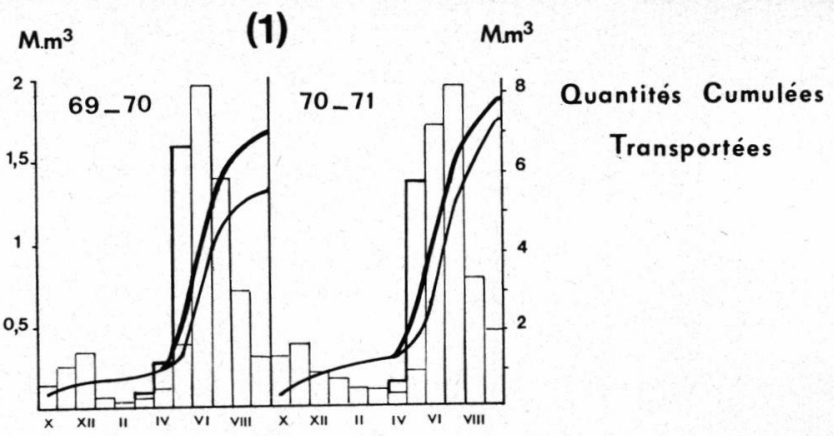

(2)

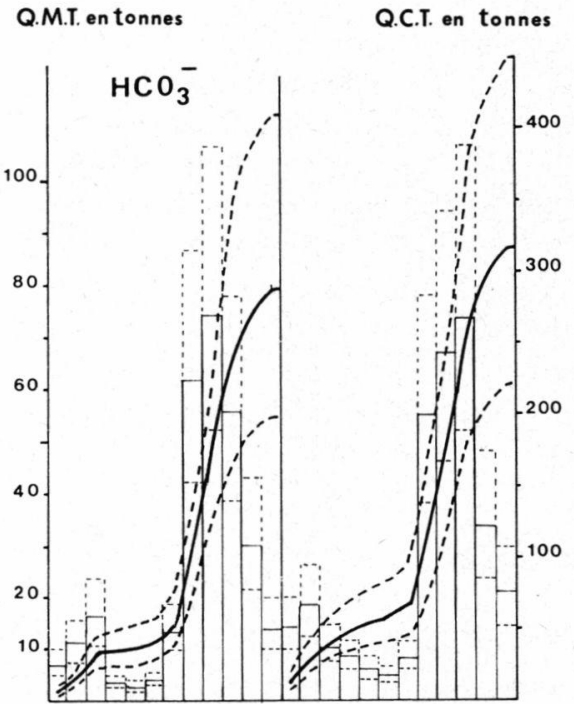

Q.M.T. t.

Q.C.T. $\mathbf{t}$.
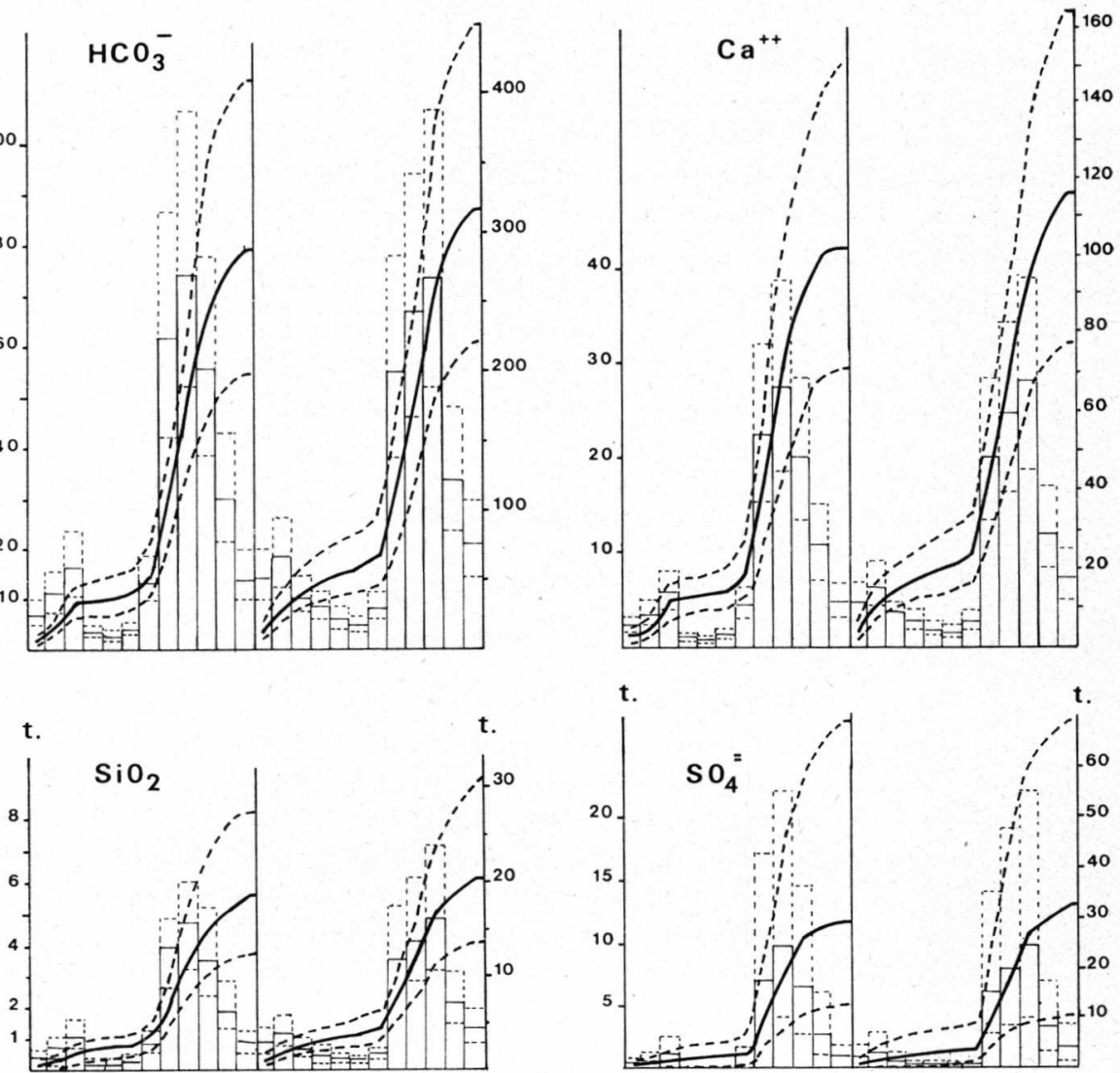

FIG. 8. - Quantités de substances dissołtes amenées au lac d'Orédon durant les deux années hydrologiques 1969-1970 et 1970-1971.

- (1) Régime hydrique à la station D (1 $850 \mathrm{~m}$ d'altitude). Les volumes d'eau mensuels écoulés (et par suite les volumes d'eau cumulés) sont calculés à partir des enregistrements effectués à la station de référence $R(2150 \mathrm{~m}$ d'altitude), en utilisant la relation présentée à la figure 7 . Ils sont figurés en traits fins. Les traits épaissis donnent les valeurs probables de la décharge au printemps : ils tiennent compte du décalage présenté par la fonte des neiges suivant l'altitude.

- (2) Quantités de substances dissoutes amenées au lac d'Orédon - les traits pleins donnent les valeurs probables - les tirets donnent les limites de confiance des estimations. 
Q.M.I. kg.

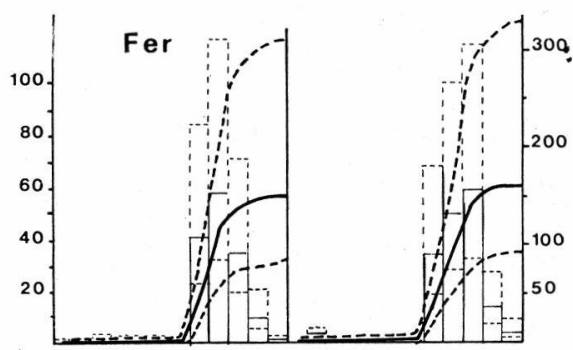

kg.

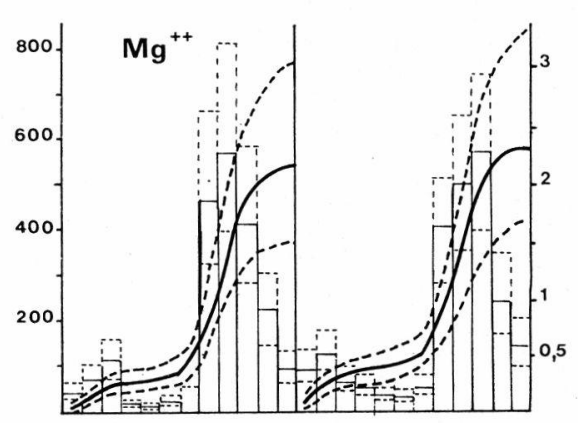

t.

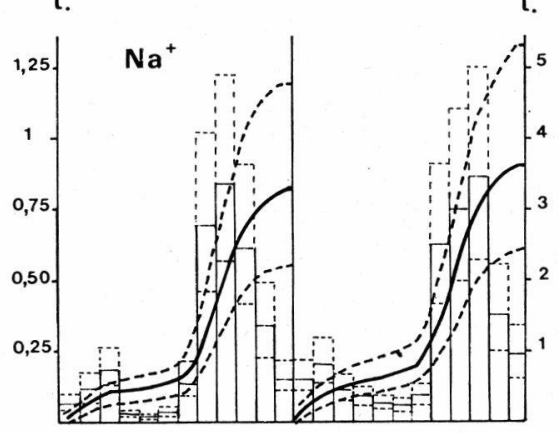

t.
Q.M.T. kg.

Q.C.T. kg.
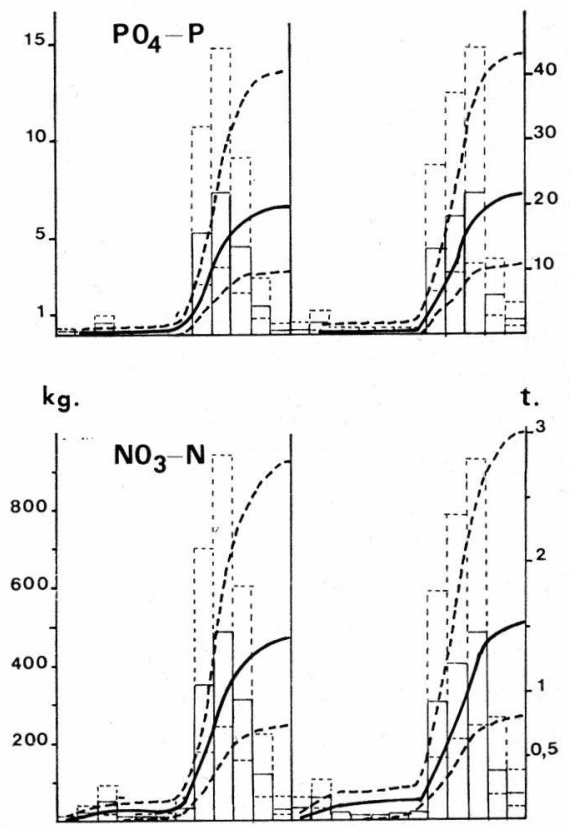

t.

t.

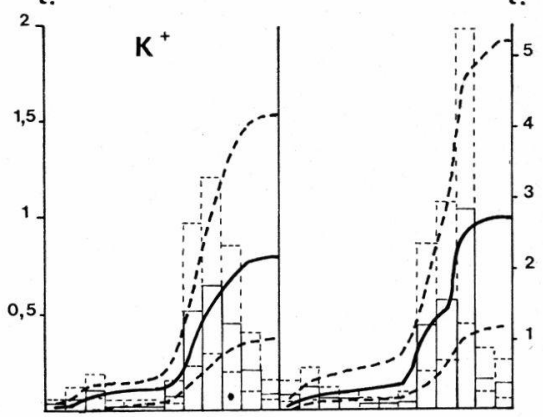

(3)
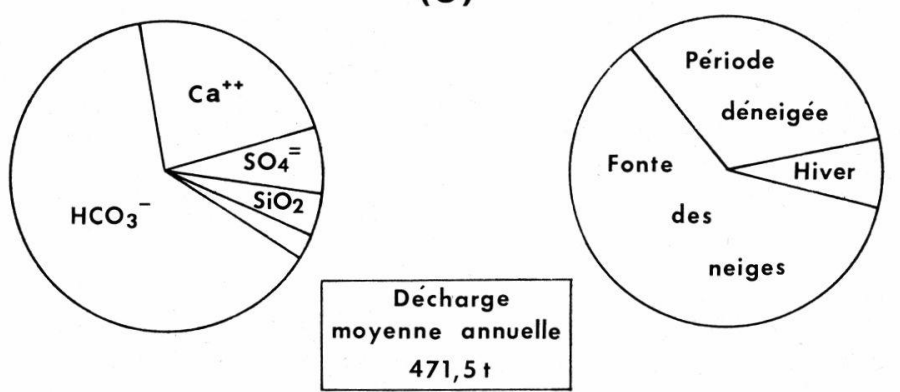

FIG. 8. - (2) Suite.

- (3) Importance comparée des saisons et des divers éléments dans la décharge moyenne annuelle. 


\subsection{2. - ORigine des SUbStances dissoutes.}

La composition chimique d'une eau résulte :

- de la nature de l'eau d'alimentation (précipitations pluvieuses ou neigeuses);

- des modifications apportées à ces caractéristiques initiales : 1) par des facteurs exogènes (géologie, pédologie, régime hydrique, évaporation) ; 2) par des influences «endogènes» (lit du cours d'eau et phénomènes biologiques qui s'y déroulent).

\subsubsection{1. - Les précipitations.}

Le tableau IX indique la composition de quelques échantillons d'eau de pluie et de neige. L’hétérogénéité de la qualité des précipilations, souvent mentionnée (Feth 1964, Gorham 1955, 1961), est illustrée par les différences importantes que présentent parfois les valeurs extrêmes. Au contact du sol, l'eau s'enrichit en sels minéraux et ses caractéristiques chimiques en un point donné, résultent alors essentiellement du trajet qu'elle a parcouru.

Tableau IX. - Composition chimique de l'eau de pluie et de neige : valeurs moyennes maximales et minimales. 7 analyses d'eau de pluie et 5 de neige ont été réalisées. Les teneurs en $\mathrm{Ca}^{++}, \mathrm{HCO}_{3}^{-}, \mathrm{SO}_{4}{ }^{-}$et $\mathrm{Cl}^{-}$sont exprimées en mg/l, les autres en $\mu \mathrm{g} / \mathrm{l}$.

\begin{tabular}{|c|c|c|c|c|c|c|c|c|c|c|c|}
\hline & . & $\mathrm{Ca}^{++}$ & $\mathrm{Mg}^{++}$ & $\mathrm{Na}^{+}$ & $\mathbf{K}^{+}$ & $\mathrm{HCO}_{3}^{-}$ & $\mathrm{PO}_{4}-\mathrm{P}$ & $\mathrm{NO}_{3}-\mathrm{N}$ & $\mathrm{Cl}^{-}$ & $\mathrm{SO}_{4}^{--}$ & $\mathrm{SiO}_{2}$ \\
\hline \multirow{3}{*}{ Neige } & $\max$ & 2 & 140 & 110 & 80 & 0,976 & 9 & 135 & 1,5 & 4,5 & 40 \\
\hline & $\overline{\mathrm{M}}$ & 1,5 & 80 & 90 & 50 & 0,488 & 4 & 80 & 1 & 2 & 30 \\
\hline & $\min$ & 0,7 & 60 & 60 & 30 & 0 & 3,2 & 0 & 0 & 0,5 & 10 \\
\hline \multirow{3}{*}{ Pluie } & $\max$ & 2 & 165 & 550 & 100 & 3,05 & 9,5 & 142 & 0,75 & 2,5 & 35 \\
\hline & $\overline{\mathbf{M}}$ & 1,2 & 130 & 380 & 80 & 1,83 & 8 & 95 & 0,5 & 2 & 29 \\
\hline & $\min$ & 0,5 & 80 & 200 & 70 & 1,22 & 5 & 60 & 0 & 1 & 15 \\
\hline
\end{tabular}

\subsubsection{2. - Le contexte géographique.}

Le vallon est essentiellement constitué par trois types de roches : 1) calcaires cristallins du dévonien et barrégiennes (calcaires à rubans siliceux métamorphiques et déformés); ils forment la muraille sud du cirque supérieur ; 2) schistes et pélites gréseuses du dévonien inférieur dominant au-dessus de $2200 \mathrm{~m}$; au niveau de la cascade du cirque d'amont les schistes et calcschistes sont 
souvent marron-rouge, couverts d'hydroxydes ferriques; 3 )granites amphiboliques du silurien (dominant au-dessous de $2200 \mathrm{~m}$ ). Ces ensembles qui présentent des zones d'éboulis grossiers (cas des granites) ou fins (cas des pélites et des schistes) sont couverts de végétation dès que la pente permet l'installation d'un sol.

C'est à partir de ces formations, des principaux minéraux des roches, de la plus ou moins grande capacité qu'elles ont de libérer les éléments minéraux, ainsi que de la mobilité relative des ions, que nous avons tenté d'apprécier l'influence du bassin versant sur le chimisme des eaux.

- Les principaux minéraux constitutifs des roches ont été déterminés par observations microscopiques de lames minces. Les données générales exposées ci-après font référence au travail de synthèse de Hem (1970).

- Le magnésium est, dans les roches éruptives, un constituant typique des minéraux ferro-magnésiens sombres, parmi lesquels amphiboles et micas sombres (biotite) sont largement répandus dans le vallon; les chlorites, minéraux d'altération de la biotite, sont une autre source magnésienne bien représentée au sein des pélites.

- Le sodium et le potassium entrent dans la composition des plagioclases, feldspaths constitutifs des roches granitiques. Notons que le sodium est en grande partie issu des précipitations.

- Le calcium est présent dans les roches éruptives, mais en Estaragne les fortes concentrations sont liées à la présence de calcite abondante dans les calcaires cristallins et les barrégiennes.

- Amphiboles et biotites sont dans le vallon les minéraux essentiels contenant du fer. Cependant, la mise en solution du fer à partir des minéraux silicatés est un processus lent et de faible intensité (Hem 1970). Dans les conditions de milieu étudié, pH légèrement basique et concentration en $\mathrm{O}_{2}$ dissous élevée, le fer est sous forme de particules d'hydroxydes ferriques ou de complexes organiques. Dans les parties hautes du vallon, les plaques marron-rouge de nombreux schistes et calcschistes témoignent de la présence d'hydroxydes ferriques. Ces formes sont généralement les sources principales dont provient le fer dissous; elles sont à l'origine des concentrations en fer total relevées sur le torrent.

- Le soufre est largement répandu sous forme de sulfures métalliques qui, oxydés, sont transformés en sulfates. Au cirque supérieur, ces formes sont particulièrement abondantes dans les rochers couverts de rouille. L'ion $\mathrm{SO}_{4}^{--}$peut constituer des associations telles que $\mathrm{NaSO}_{4}^{-}$et $\mathrm{CaSO}_{4}^{-}$. Il annexe alors une partie des ions $\mathrm{Ca}^{++}$et joue donc indirectement un rôle dans les possibilités de formation des bicarbonates évoquées précédemment. A la station A, 
le calcium présente avec l'alcalinité une corrélation moins forte qu'au niveau du lac; d'autre part, la pente de la droite relative au calcium, qui traduit l'évolution des concentrations en calcium en fonction des débits, est plus proche de celle des sulfates que de celle des bicarbonates.

- Le phosphore est un constituant essentiel de l'apatite, minéral décelé dans les calcaires. Cependant, et contrairement à la majorité des éléments étudiés ci-dessus, les précipitations atmosphériques constituent certainement l'origine principale des teneurs observées. Les concentrations trouvées dans l'eau de pluie ou de neige sont le plus souvent supérieures aux teneurs maximales relevées sur le terrain.

- Les sources d'azote généralement reconnues sont l'atmosphère, les composés azotés d'érosion continentale, le sol, la dégradation des matières organiques. En Estaragne, les précipitations semblent à cet égard de peu d'importance. Les analyses de neige, dè pluie, révèlent en effet des teneurs de l'ordre de $100 \mu \mathrm{g} / \mathrm{l}$ de $\mathrm{NO}_{3}-\mathrm{N}$. Le rôle des roches n'est pas prépondérant; ainsi Feth (1964) a montré que si les données de Wlotslka (1961) sont applicables dans son étude de la Sierra Nevada, le granite ne peut y être assimilé à une source importante de nitrates; nous verrons par ailleurs que nos roches ne semblent pas capables de libérer des quantités mesurables d'azote. L'activité biologique et la décomposition des substances organiques sont, par contre, considérées comme les facteurs essentiels de l'enrichissement en nitrates de l'eau; il est done important d'envisager sur l'ensemble du vallon, l'apport azoté des excréments de mouton en pâturage estival.

Au cirque supérieur, l'humus noir et les tourbières à Hypnacées paraissent déterminer en grande partie les teneurs en $\mathrm{NO}_{3}-\mathrm{N}$ enregistrées. Les ruisselets qui parcourent ces tourbières présentent des concentrations de l'ordre de $190 \mu \mathrm{g} / \mathrm{l}$. Breuillaud et Grizou (1961) ont d'autre part montré que dans ces zones tourbeuses, protéolyse et dénitrification sont importantes, malgré une activité bactérienne faible.

Dans la partie moyenne du vallon se développe une prairie subalpine. La végétation est généralement source de nitrates, mais dans ce milieu, la dégradation végétale est particulièrement lente, du fait de la nature même des végétaux (aiguilles de pins amenées par le vent, tiges el feuilles de graminées). En outre, les nitrates constituent pour la végétation en place un ion essentiel à la croissance. Ces deux phénomènes conjugués peuvent être responsables de la pauvreté relative des affluents (excepté a4 issu d'une tour- 
bière). Ainsi les produits décomposés sont peu abondants et ne s'accumulent pas, d'autant qu'en phase déneigée, les pluies violentes drainent périodiquement au torrent les maigres quantités dégradées et les herbes mortes avant le début de leur décomposition.

Le relèvement des concentrations enregistrées en aval, peut être mis en relation avec la végétation plus dense de hêtres et de sapins, un sol plus profond et des produits de décomposition plus abondants qu'aux altitudes supérieures.

- Nous avons tenté d'apprécier, par des expériences simples, la solubilité des différents substrats minéraux. Quelques échantillons des principaux types de roches dominantes sont plongés dans un même volume d'eau distillée; celle-ci est agitée pendant la durée de l'expérience ( $5 \mathrm{mn}$ à 1 jour) puis analysée; les résultats sont ramenés à des surfaces identiques de roches. Les roches sont classées selon la faculté décroissante qu'elles ont de libérer l'élément considéré :

- Silice : granites, quartzites et pélites, schistes, barrégiennes, calcaires;

- Sulfates : quartzites et pélites, barrégiennes, schistes, calcaires, granites;

- Ca: barrégiennes et calcaires, quartzites et pélites, schistes et granites;

- $\mathrm{Na}$ : granites, barrégiennes et calcaires, schistes, quartzites et pélites;

- K : granites, barrégiennes, calcaires et schistes, quartzites et pélites;

- Mg : granites, barrégiennes, calcaires et schistes, quartzites et pélites;

- Fe : quartzites et pélites, barrégiennes, schistes, calcaires, granites;

- $\mathrm{PO}_{4}-\mathrm{P}$ : schistes, granites et calcaires;

- $\mathrm{NO}_{3}-\mathrm{N}$ : pas de quantités décelables.

La nature géologique des substrats et la possibilité qu'ils ont de libérer leurs constituants permet de comprendre et d'expliquer en grande partie l'évolution des composantes chimiques de la source à l'embouchure.

Ainsi le calcium, les sulfates et le fer sont essentiellement libérés par les pélites, les calcschistes et les calcaires métamorphiques qui dominent en altitude. Leurs concentrations diminuent jusqu'au lac et les quantités charriées augmentent peu. Par contre, la silice, le sodium, le potassium et le magnésium sont issus des différents types de roches et particulièrement du granite; leurs concentrations moyennes sont stables tout au long du cours, et leur transport augmente nettement de l'amont vers l'aval. Ces éléments n'apparaissent cependant qu'en quantité limitée car l'altération des roches cristallines est faible.

- L'étude des nitrates et des phosphates met toutefois en évidence les limites d'une interprétation, qui ne tiendrait compte que 
des observations développées ci-dessus. Sur le bassin versant entrent en jeu des phénomènes complexes, tels que : l'influence de la végétation qui ralentit l'érosion (Bormann et al. 1968) et est à la fois utilisatrice et source de sels minéraux ; les processus d'adsorption au niveau des argiles; la durée de rétention de l'eau qui varie selon les terrains, etc.

Une approche globale de ces phénomènes a été réalisée pour quelques éléments, par l'étude du rapport : \% de l'élément dans l'eau/\% de l'élément dans la roche. Ce rapport donne une image de la mobilité apparente relative des ions (Smyth 1913, Feth 1964).

La séquence $\mathrm{Ca}>\mathrm{Na}>\mathrm{Mg}>\mathrm{K}>$ Silice $>$ Fer est obtenue au bas du cours principal, donc pour un bassin versant qui intègre les divers types de roches. Elle est analogue à celle rapportée par Feth (1964) pour les cours d'eau et les roches granitiques de la Sierra Nevada et conduit à des conclusions de même type.

- Le calcium est l'élément le plus mobile, suivi de loin par le sodium et le magnésium. L'ordre de ces deux derniers ions est inversé si l'on tient compte de l'apport ionique initial des précipitations.

- Le fer est l'élément le moins mobile; dans les conditions de milieu il reste sur place sous forme d'hydroxydes.

- La silice et le potassium ont des mobilités voisines et faibles. Dans le vallon, la silice est essentiellement sous forme de quartz, minéral particulièrement résistant à l'altération chimique. $\mathrm{K}^{+}$est nécessaire à la croissance de la végétation qui l'utilise. Il est en outre retenu avec la silice au niveau des argiles.

Les rapports établis pour ces six éléments à la suite des expériences décrites ci-dessus, présentent un classement qui diffère essentiellement du précédent par la position du potassium. $\mathrm{K}$ se place au deuxième ou au troisième rang, toujours avant $\mathrm{Mg}$. Cette différence entre les «séquences expérimentales» et les séquences déduites des analyses effectuées en Estaragne traduit bien la «capture » du potassium par le bassin versant.

\subsubsection{3. - Le lit du cours d'eau.}

Il est principalement constitué de pélites, de schistes et de calcaires, le granite formant soit un sable grossier qui s'accumule en l'absence de courant, soit d'imposants blocs au centre du lit ou sur les berges. L'eau attaque ces roches et, selon la durée et l'importance du contact, s'enrichit en sels minéraux. Les résultats d'une expérience du même type que celles décrites ci-dessus sont présentés dans la figure 9 . 
Elle montre l'enrichissement en $\mathrm{Ca}^{++}$et en $\mathrm{Na}^{+}$de deux volumes d'eau V1 et V2: $\left(\mathrm{V}_{12}=4 \mathrm{~V}_{1}\right)$ au contact d'un substrat calcaroschisteux. L'augmentation du volume d'eau correspond en fait à une réduction des possibilités de contact avec le substrat. L'enrichissement diminue quand la quantité d'eau augmente. Cette expérience schématise une augmentation de débit du torrent. Or, en Estaragne, lorsque l'écoulement est multiplié par quatre, la durée du trajet de la source à l'embouchure (donc la durée du contact avec le lit) est divisée par deux. L'influence du substrat en période d'étiage et lors d'une crue qui déterminerait un écoulement quatre fois supérieur, peut être envisagée en comparant dans la figure 9, le contenu en sels minéraux du volume $V 1$, à un temps $T$, avec celui présenté par le volume $\mathrm{V} 2$, à un temps $\mathrm{T} / 2$.

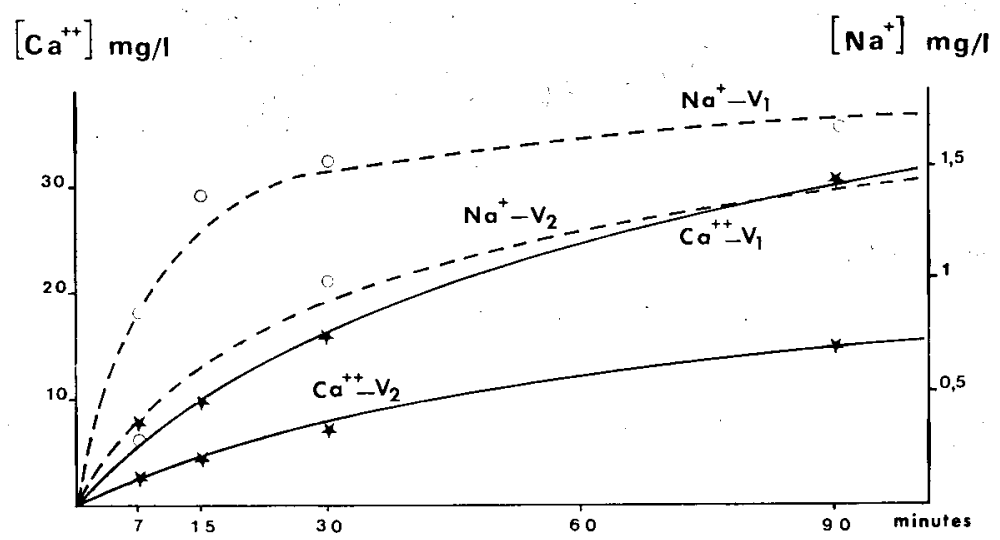

Fig. 9. - Evolution en fonction du temps et du volume d'eau des quantités de $\mathrm{Ca}^{++}$(traits continus) et de $\mathrm{Na}^{+}$(tirets) libérées par un mélange de calcaires, de schistes et de pélites. Deux volumes d'eau différents V1 et V2 (V2 $=4 \times V 1)$ sont utilisés pour chaque élément.

De la même façon, les détritus qui s'amoncellent derrière les blocs et sous les gros galets, peuvent constituer une source d'ions permanente (cf. Thomas 1970, Hynes et Kaushik 1969). Le rôle de ces détritus, accumulés en quantité surprenante vu les conditions d'écoulement, est toutefois limité. Le maintien en hiver, des fortes teneurs en $\mathrm{O}_{2}$, montre que la dégradation de ces substances est faible et qu'elle n'est absolument pas comparable à celles observées par Schneller (1955) ou Slack (1964).

Etant donné la faible minéralisation de l'eau, l'enrichissement au contact du substrat n'est pas à négliger, bien que ses conséquences soient certainement limitées par la brièveté du parcours amont - aval (moins d'une heure en période de crue, cinq à six heures à l'étiage). 
Les quantités de matériel charrié augmentent généralement de l'amont vers l'aval du fait des apports des affluents et de l'eau de ruissellement. Seuls les premiers sont mesurables. La charge des affluents constitue donc l'apport minimal envisageable. Or entre deux stations consécutives, cet apport est souvent supérieur à l'augmentation des quantités transportées. Si on exclut d'éventuels échanges avec un sous-écoulement pauvre en substances dissoutes, ce fait ne peut résulter que de processus biologiques.

Le rôle des communautés vivantes sur les concentrations en sels dissous (essentiellement $\mathrm{NO}_{3}-\mathrm{N}, \mathrm{PO}_{4}-\mathrm{P}, \mathrm{SiO}_{2}$ ) a été maintes fois évoqué (cf. Hynes 1970, Ladle et Casey 1972, Serra Tosio 1969, Egborge 1971, Rigler 1961, Bombowna 1965, Casey et Newton 1972, Capblancq 1972, Bordes, Luchetta el Rochard 1973 ...). Leur influence dépend de l'état physiologique des communautés, c'està-dire de l'antagonisme biosynthèse-biodégradation. Dans cette optique le tableau $\mathrm{X}$ donne : 1 ) la composition chimique de l'eau en amont et en aval d'une zone d'une cinquantaine de mètres de longueur, abondamment couverte d'hydrures âgées ; 2 ) le contenu en cations de touffes d'hydrures peuplées de larves de Chironomides récemment écloses. Cette dernière analyse met nettement en

TABleau $X$. - Influence de la végétation benthique sur les propriétés chimiques de l'eau :

I. Composition chimique de l'eau avant son passage sur des hydrures.

II. Après son passage sur les hydrures.

III. Composition cationique de touffes d'hydrures colonisées par des Chironomides.

* Alcalinité, Silice, Chlorure, Calcium et Sulfates sont exprimés en $\mathrm{mg} / \mathrm{l}$, les autres en $\mu \mathrm{g} / \mathrm{l}$.

* Valeurs en $\mathrm{mg} / \mathrm{g}$ de poids sec.

\begin{tabular}{lrlllllllllll}
\hline & $\mathrm{K} \mathrm{20}^{\circ}$ & $\mathrm{Ca}^{++}$ & $\mathrm{Mg}^{++}$ & $\mathrm{Na}^{+}$ & $\mathrm{K}^{+}$ & $\mathrm{HCO}_{3}{ }^{-}$ & $\mathrm{PO}_{4}-\mathrm{P}$ & $\mathrm{NO}_{3}-\mathrm{N}$ & $\mathrm{Cl}^{-}$ & $\mathrm{SO}_{4}^{--}$ & $\mathrm{SiO}_{2}$ & $\mathrm{Fe}^{++}$ \\
\hline * I & 100,2 & 17,1 & 210 & 410 & 250 & 50,51 & 4,2 & 210 & $\mathbf{0 , 8 5}$ & 4,9 & 1,8 & 0 \\
* II & 97,8 & 17,25 & 190 & 400 & 240 & 50,75 & 4,9 & 280 & 0,75 & 3,8 & 1,95 & traces \\
* III & & 52,6 & 20 & 12,8 & 43,8 & & & & & & & \\
\hline
\end{tabular}

évidence l'impact, que réalisent directement ou indirectement les communautés vivantes sur le contenu ionique de l'eau. Notons que par rapport aux concentrations trouvées dans le milieu liquide, le potassium est le cation le plus abondant dans les hydrures.

L'utilisation de cet élément par la végétation, tant sur le bassin versant que dans le cours d'eau lui-même, est certainement responsable de l'absence de relations nettes entre les concentrations en potassium et les débits.

Nous avons d'autre part rattaché l'irrégularité de l'évolution altitudinale des phosphates au développement des algues sur le torrent. 


\section{2. - Evolution des composantes chimiques en fonction du débit.}

Les variations que nous avons notées sont en général analogues à celles rapportées dans la littérature (Fgborge 1971, Minschall 1968, Crisp 1966, Casey et Newton 1972, Holden et Green 1960, Rzóska et Talling 1966, Bishop 1973). La conductivité, les teneurs en calcium et en magnésium diminuent quand le débit augmente. L'alcalinilé évolue généralement de même, mais une photosynthèse intense peut inverser cette relation en période d'étiage (Egborge 1971). L'évolution des teneurs en orthophosphates est plus variable : alors que les concentrations sont généralement déterminées par l'influence humaine et les phénomènes biologiques, la corrélation positive que nous avons trouvée en Estaragne semble essentiellement due à la charge initiale de l'eau de pluie.

Le rapport existant entre le chimisme du torrent et son débit, ainsi que les différences de détail présentées par celte relation, se trouvent résumés au cours d'un cycle journalier, lors de la fonte des neiges. A cette époque, le torrent accuse des fluctuations hydriques quotidiennes consécutives à la fusion nivale; la composition chimique de l'eau varie selon l'importance du débit. La figure $\mathbf{1 0}$ indique les valeurs réelles trouvées lors de cinq analyses réalisées à différentes heures d'une journée printanière. Elle montre, en outre, l'évolution théorique des concentrations, calculées à l'aide des corrélations générales «débit probable - concentration probable ».

- Les valeurs mesurées et les valeurs calculées dessinent une évolution parallèle.

- Les analyses révèlent généralement des valeurs proches de la courbe, souvent situées au-dessous lors de la montée des eaux, alors que les teneurs sont supérieures aux moyennes théoriques pendant les décrues.

Ces écarts reflètent les deux phases de la crue nivale :

* l'augmentation du débit est tardive et rapide : le matin, l'eau de neige imbibe le bassin versant; plus tard elle ruisselle rapidement sur le sol gorgé d'eau et se charge peu en sels minéraux avant de rejoindre le torrent;

* la périod a de décroissance des débits est prolongée; l'eau retenue par le bassin versant s'écoule lentement; elle acquiert une composition chimique relativement riche, comparable à celle observée après un orage.

\section{3. - Quantités transportées.}

La masse de substances dissoutes charriées par le torrent, intègre l'ensemble des observations formulées. De plus, il est possible d'utiliser l'estimation des quantités transportées faite à chacune 

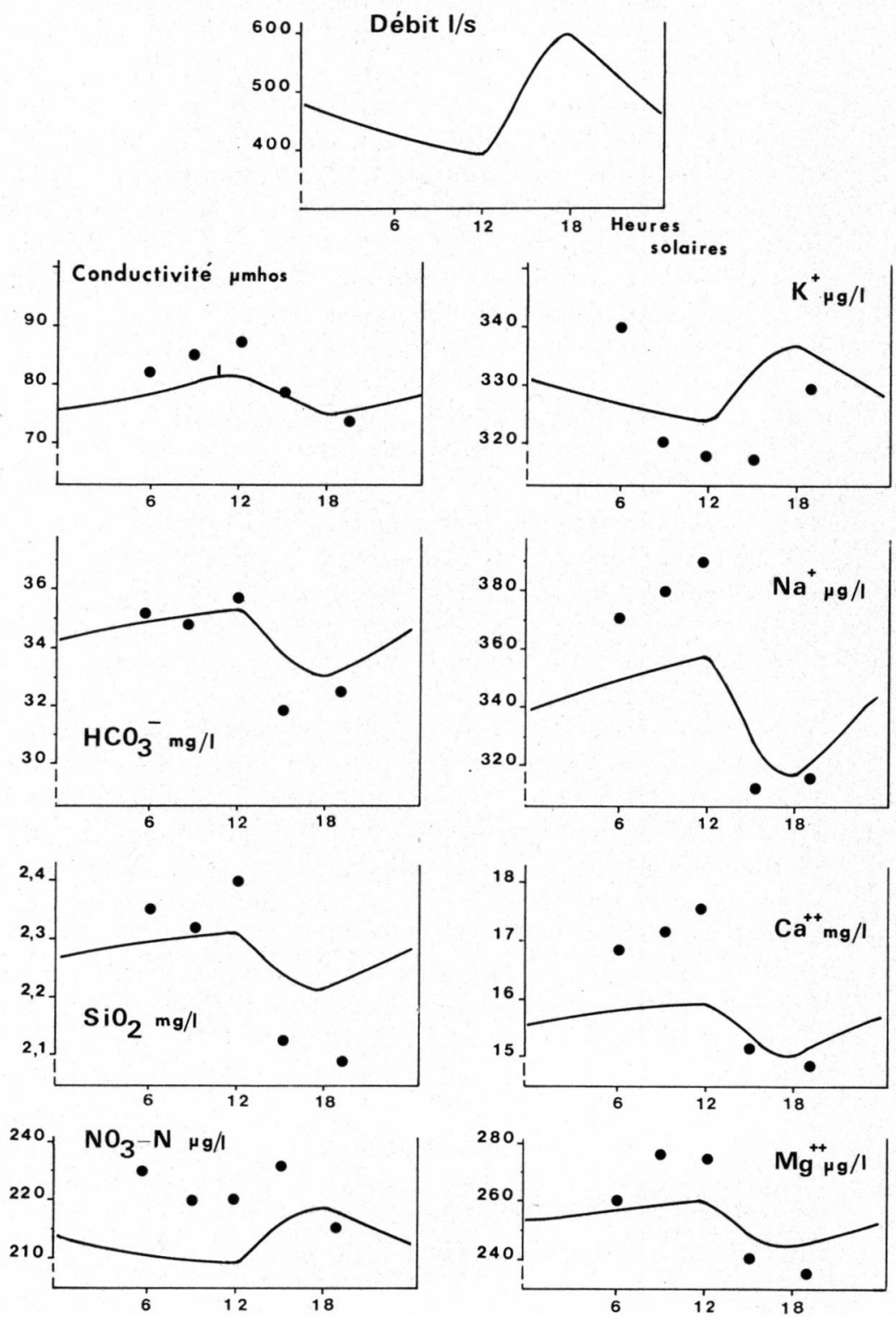

FIG. 10. - Variations quotidiennes de la composition chimique de l'eau pendant la fonte des neiges (station $R$ ). Les points indiquent les valeurs résultant des cinq dosages effectués au cours d'une journée. La courbe donne l'évolution théorique de la composition chimique calculée en fonction du débit. 
des stations du cours principal pour déterminer ce qu'apporte chaque $\mathbf{k m}^{2}$ de bassin versant. Les apports respectifs de territoires choisis dans des terrains de nature géologique différente peuvent être comparés s'ils sont évalués par unité de volume d'eau de drainage. Il est ainsi possible de comparer, par exemple, l'apport de $1 \mathrm{~km}^{2}$ de la surface «B - A 》 (territoire correspondant à la différence de surface des bassins versants à la station $A$ et à la station B) formée de schistes, de pélites et de calcaires, et de $1 \mathbf{k m}^{2}$ de la surface $~ C \mathrm{C}-\mathrm{R}$ » essentiellement granitique. Pour une même étendue, les territoires «B - A $\gg$ apportent plus de calcium et de sulfates que ceux situés entre les stations $C$ et $R$. Ces derniers, en revanche, libèrent plus de sodium, magnésium, potassium et silice. Une approche semblable $d u$ rôle du bassin versant peut être généralisée à des ensembles homogènes d'un point de vue pédologique ou géologique. L'interprétation doit néanmoins rester prudente, compte tenu de sous-évaluations importantes que peuvent engendrer les eaux d'infiltration.

La décharge amenée au lac d'Orédon lors des deux années hydrologiques étudiées a été calculée de façon indirecte. Toutefois la validité de l'estimation des deux facteurs du produit débit concentration est prouvée par les analyses effectuées en 1970 à Ia station D. Leurs résultats ( $f i g .11$ ), sont toujours compris entre les limites de confiance des valeurs calculées d'après les débits enregistrés, aux dates de prélèvement, à la station de référence $R$.

Cette décharge est essentiellement déterminée par le régime hydrologique : plus de $65 \%$ des substances dissoutes sont charriées lors de la fonte des neiges. Ce phénomène est général dans les cours d'eau qui présentent de fortes variations hydriques saisonnières, même lorsque les concentrations varient en raison inverse du débit (Hynes 1970).

Les quantités transportées en phase déneigée sont modestes. Cependant, dans un système montagnard, la saison estivale est fondamentale. C'est en effet la période essentielle de croissance des communautés torrenticoles végétales et animales. Les invertébrés utilisent pour leur croissance des sels minéraux, qu'ils stockent dans leurs tissus. Ils rejettent en outre, à chaque mue, une exuvie peu dégradable qui sera charriée plus ou moins rapidement dans le lac. Les végétaux, qui accumulent en grande quantité les substances minérales, doivent évidemment être considérés lors de l'établissement de bilans chimiques saisonniers ou annuels. De telles estimations permettront d'apprécier l'importance relative des communautés dans l'écosystème et de préciser son fonctionnement. 


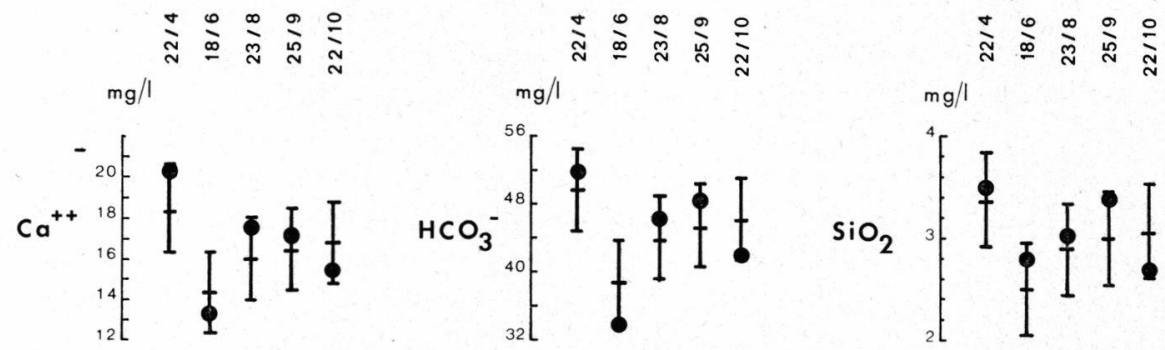

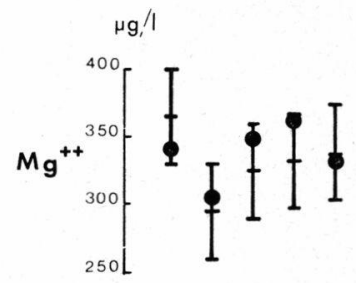

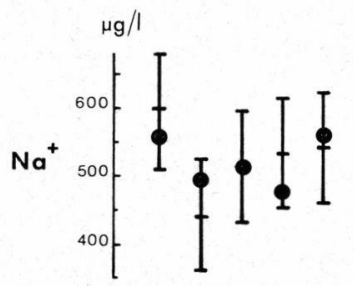

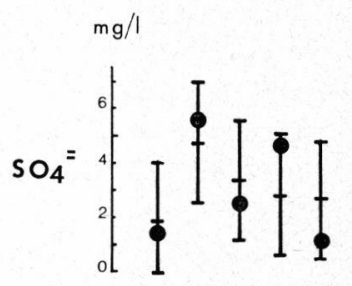

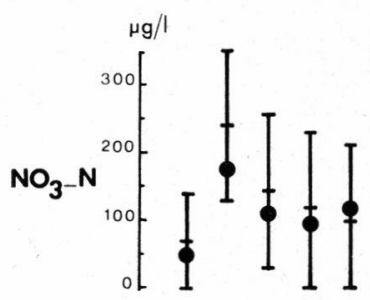

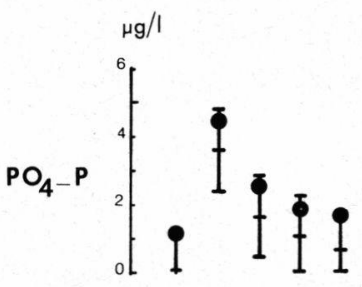

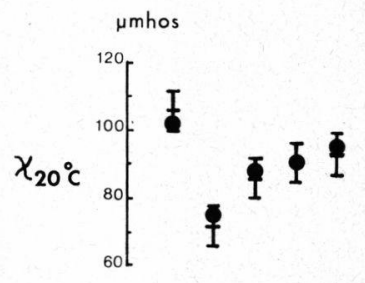

Fig. 11. - Fiabilité des estimations à la station D. Les points indiquent les valeurs réelles obtenues par dosages d'échantillons prélevés à la station D en 1970. Les traits indiquent les valeurs probables, maximales et minimales calculées à chaque date de prélèvement à partir : 1) des enregistrements de débits effectués cette année-là à la station $R ; 2$ ) des données recueillies durant l'été 1972.

\section{RESUME - CONCLUSION}

L'étude chimique du torrent d'Estaragne et des ses affluents permet de dégager plusieurs points essentiels.

- La conductivité électrique de l'eau, à $20^{\circ} \mathrm{C}$, est de l'ordre de $40 \mu$ mhos sur les terrains granitiques, de $110 \mu$ mhos sur les formations pélitiques et calcschisteuses. La composition chimique est caractérisée par l'importance des bicarbonates et du calcium, qui représentent plus de $90 \%$ du contenu ionique de l'eau.

- Les précitations semblent déterminer en grande partie les teneurs en $\mathrm{PO}_{4}-\mathrm{P}$ et à un degré moindre celles en $\mathrm{Na}^{+}$. 
-- Les teneurs de la plupart des éléments étudiés diminuent de l'amont vers l'aval; cette évolution est déterminée par la structure du bassin versant dont le rôle sur la composition chimique de l'eau peut se résumer ainsi :

* Au cirque supérieur d'Estaragne, les principaux types de roches du vallon coexistent; à ce niveau, le torrent se forme par la confluence de petites sources, de ruisselets à écoulement lent, d'eau d'infiltration. L'eau, de composition chimique diversifiée, est relativement riche en éléments dissous.

* Vers l'aval, les roches calcaires altérables, ou les éléments de schistes et de pélites désagrégés qui retiennent sur les pentes l'eau des précipitations, font place à des roches éruptives cristallines, peu altérables, qui ne libèrent que de faibles quantités ioniques.

Les conséquences de cette hétérogénéité du substrat sont accentuées par la succession altitudinale des sols.

* Sur les sommets et les versants abrupts, la roche mère est nue, soumise à une érosion physique intense. L'altération superficielle est forte et se traduit par une libération importante de substances minérales.

* Plus bas, sur les replats, se développe une végétation à fétuques qui utilise pour sa croissance les sels minéraux issus de l'altération. Les basses températures ne permettant qu'une décomposition faible, c'est l'utilisation des sels qui est le phénomène dominant. La présence de végétation ralentit en outre l'érosion en favorisant la rétention de l'eau et l'évaporation. La minéralisation de l'eau qui rejoint le torrent, sera donc comparativement plus faible que dans les zones dénudées du cirque supérieur.

* Avec la hêtraie sapinière qui borde les derniers cent mètres du torrent, une autre phase de l'évolution est abordée. La végétation est plus dense, le sol plus évolué. Quelques éléments sont relâchés et leur concentration augmente légèrement dans le torrent.

-.. Les composantes chimiques - à l'exception de $\mathrm{K}^{+}$— présentent en général à toutes les stations, une corrélation significative avec le débit. Ces corrélations, dont on a vérifié la validité, sont utilisables pour estimer les quantités de matériel charrié et pour préciser le rôle des affluents et des divers territoires du bassin versant dans ce transport. Les $3 / 4$ des substances dissoutes amenées au lac proviennent du tiers supérieur du vallon.

- Le calcul des décharges mensuelles met en évidence l’importance fondamentale de l'écoulement à la fonte des neiges. Bien que 
les éléments dominants $\left(\mathrm{Ca}^{++}\right.$et $\left.\mathrm{HCO}_{3}{ }^{-}\right)$présentent des corrélations négatives avec les débits, plus de $65 \%$ de la décharge annuelle s'effectue pendant cette période de hautes eaux.

- Les quantités de substances minérales dissoutes déversées annuellement dans le lac d'Orédon ont été estimées à 451 tonnes en 1970 et à 497 tonnes en 1971 .

\section{TRAVAOX CITES}

ApHA. 1960. - Standard methods for the examination of water and wastewater including bottom sediments and sludges. $13^{\mathrm{e}}$ édit. 874 p. Washington.

Barkère (P.). 1952. - Le relief des massifs granitiques du Néouvielle, de Cauterets et de Panticosa, Revue géogr. Pyrénées S.-O., $23: 69$ $98,+2 \mathrm{pl}$.

Bishop (J. E.). 1973. - Limnology of a small Malayan River, Sungai Gombak. sunk, The Hague, 485 p.

Bombówna (M.). 1965. - The chemical composition of the water of streams of the Polish high Tatre Mts, particulary with regard to the stream Sucha Woda. Acta hydrobiol., Krakow, 13 (4) : 379-391.

Bordes (J. M.), Luccheta (J. C.) et Rochard (M.) 1973. - Etude d'un écosystème d'eau courante : le Lot. Thèse $3^{\circ}$ cycle, U.P.S., Toulouse.

Bormane (F. H.), Lickens (G.E.), Fischer (D. W.), Pierce (R. S.) 1968. - Nutrient loss accelerated by clearcutting of a forest ecosystem. Science, N. Y., 159 : 882-884.

Breuilatud (J.) el Grizou (V.). 1961. - Etude microbiologique d'un sol tourbeux de montagne. Bull. Soc. Hist. Nat. Toulouse, $96(3 / 4)$ : 275-280.

Capbianco (J.) et Laville (H.). 1968. - Etude morphométrique et physico-chimique de neuf lacs du Massif de Néouvielle (HautesPyrénées). Annls Limnol., 4 (3) : 275-324.

Casey (H.) et Newton (P.V.R.). 1972. - The chemical composition and flow of the South Winterbourne in Dorset. Freshwater Biology, $2(3)$ : 229-234.

Casey (H.) et Newton (P.V.R.). 1973. - The chemical composition and flow of the River Frome and its main tributaries. Freshwater Biology, 3 (4) : 317-334.

CRisp (D. T.). 1966. - Input and output of minerals for an area of pennine Moorland : the importance of precipitation, drainage, peat erosion and animals, $J$. appl. Ecol., 3 : 327-348.

Dussart (B.). 1966. - Limnologie : l'étude des eaux continentales. 678 p. Paris.

Egborge (A.B.M.). 1971. - The chemical hydrology of the river Oshun, Wester State, Nigeria. Freshwater Biol., 1 (3) : 257-271.

Fетн (J. H.). 1964. - Sources of mineral constituents in water from Rocks Sierra Nevada, California and Nevada. Geological survey water-supply, paper $1535-\mathrm{I}$.

Golterman (H. L.). 1969. - Methods for chemical analysis of fresh waters. I.B.P. Handbook, $\mathrm{n}^{\circ} 8$.

Gormam (E.). 1955. - On the acidity and salinity of rain. Geochim. Cosmochim. Acta, 7 : 231-239. 
HEM (J. D.). 1970. - Study and interpretation of the chemical characteristics of natural water. U.S. Geol. Survey Water. Supply Paper, $1473,363 \mathrm{p}$.

Holden (M.J.) et Green (J.). 1960. - The hydrology and plankton of the river Sokoto. J. Anim. Ecol., $29: 65-84$.

Hutchinson (G. E.). 1957. - A treatise on limnology, Bd 1: 1-1015. Geography, physics and chemistry. J. Wiley \& Sons, Inc. N.Y., Chapman \& Hall, Ltd. London.

Hynes (H. B. N.). 1970. - The ecology of running waters. Liverpool University Press. 555 p.

Hynes (H. B. N.) et KaushiK (N. K.). 1969. - The relationship between dissolved nutrient salts and protein production in submerged automnal leaves. Verh. int. Verein. theor. angew. Limnol., 17 : 95-103.

LADLE (M.) et CASEY (H.). 1972. - Growth and nutrient relationships of Ranunculus penicillatus var. Calcareus in a small chalk stream. Proc. Eur. Weed. Res. Coun. 3rd int. symp. aquatic weeds, 1971 : 53-64.

Lavandier (P.). 1974. - Ecologie d'un torrent pyrénéen de haute montagne. I. Caractéristiques physiques, Annls Limnol., 10(2) : 173-219.

Minschall (G. W.). 1968. - Community dynamics of the benthic fauna in a woodland springbrook. Hydrobiologia, $32: 305-339$.

Rigler (F. H.) . 1961. - The uptake and release of inorganic phosphorus by Daphnia magna Straus. Limnol. Oceanogr., 6 : 165-174.

RJóska (J.) et Talling (J. F.). 1966. - The development of plankton in relation to hydrological regime in the Blue Nile. J. Anim. Ecol., $55: 637-662$.

Rodier (J.). 1966. - L'analyse chimique et physico-chimique de l'eau,

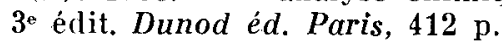

RutTner (F.). 1953. - Fundamentals of limnology. Univ. of Toronto Press, 242 p.

Saruhashi (K.). 1955. - On the equilibrium concentration of carbonic acid substances in natural water. Pap. Met. Geophys., Tokyo, $6: 39-55$.

Saunders (G.W.), Trama (F. B.) et Bachmann (R.W.). 1962. - Evaluation of a modified $\mathrm{C}_{74}$ technique for shipboard estimation of photosynthesis in large lakes. Gt lakes Res. Div., 8 1-61.

Schneller (M.V.). 1955. - Oxygen depletion in Salt Greek, indiana. Invest. indiana Lakes streams, $4: 163-75$.

Serra-Tosio (B.). 1969. - Mise en évidence, dans les cours d'eau de montagne, de variations nycthémérales de certains facteurs chimiques sous l'influence des organismes benthiques. C. R. Ac. Sc. Paris, 269 : 2 431-2 434.

SLACK (K.V.). 1964. - Effect of tree leaves on water quality in the Cacapon River, West Virginia. Prof. Pap. U.S. Geol. Surv., 475 D: 181-185.

Sмyтн (C. H., Jr.). 1913, - The relative solubilities of the chemical constituents of rocks. Jour. Geology, 21 (2) : 105-120.

Strickiand (J.D. H.) et Parsons (T. R.). 1960. - A manual of sea water analysis. Fish. Res. Bd. Can., $125: 185$ p.

Talding (J.F.). 1958. - The longitudinal succession of the water characteristics in the white Nile. Hydrobiologia $11: 73-89$.

Thomas (W. A.). 1970. - Weight and calcium losses from decomposing tree leaves on land and in water. J. Appl. Ecol., $7: 237-241$.

Wlotzka (F.). 1961. - Untersuchungen zur Geochemie des Stickstoffs : Geochim. Cosmochim. Acta., 24 : 106-154. 
Sources

Alt . 2380_2450 m

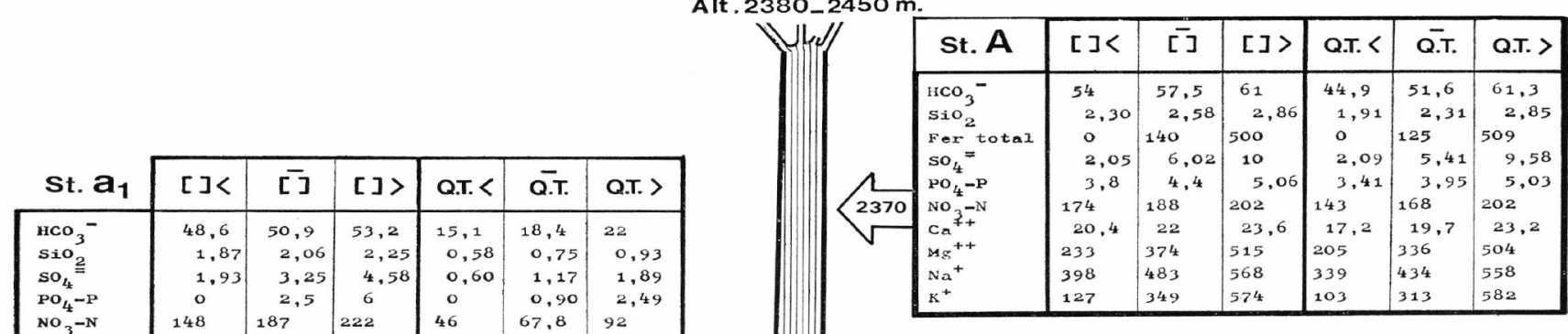

\begin{tabular}{|c|c|c|c|c|c|c|}
\hline st. $a_{3}$ & {[]$<$} & {$[\overline{[}]$} & {[]$>$} & QT. < & $\overline{\mathbf{Q T}}$. & Q.T. > \\
\hline $\mathrm{HCO}_{3}{ }^{-}$ & 39,8 & 44 & 47,1 & 2,15 & 3,16 & 4,21 \\
\hline $\mathrm{SiO}_{2}^{3}$ & 1,8 & 2,6 & 3,4 & 0,10 & 0,19 & 0,30 \\
\hline $\mathrm{SO}_{4}=$ & 1,15 & 3,35 & 6,15 & 0,05 & 0,22 & 0,51 \\
\hline $\mathrm{PO}_{4}-\mathrm{P}$ & 1,3 & 3,5 & 6 & 0,09 & 0,24 & 0,41 \\
\hline $\mathrm{NO}_{3}-\mathrm{N}$ & 95 & 164 & 225 & 4,14 & 10,3 & 18,1 \\
\hline $\mathrm{Ca}^{7+}$ & 12 & 13 & 15 & 0,67 & 1,03 & 1,46 \\
\hline $\mathrm{Mg}^{++}$ & 160 & 243 & 320 & 9,33 & 18,1 & 29,3 \\
\hline $\mathrm{Na}^{+}$ & 390 & 550 & 710 & 20,73 & 38,7 & 62,2 \\
\hline $\mathrm{K}^{+}$ & 120 & 350 & 720 & 5,18 & 22,8 & 60,5 \\
\hline
\end{tabular}

\begin{tabular}{|c|c|c|c|c|c|c|}
\hline St. $a_{4}$ & {[]$<$} & $\overline{[j}$ & {[]$>$} & Q.T. < & $\overline{\mathbf{Q} . T}$ & Q.T. > \\
\hline $\mathrm{HCO}_{3}{ }^{-}$ & 23 & 27,5 & 31,5 & 1,26 & 5 & 2,84 \\
\hline $\begin{array}{l}\mathrm{SiO}_{2} \\
\text { Fex total }\end{array}$ & $\begin{array}{l}2,22 \\
5\end{array}$ & $\begin{array}{r}3,15 \\
33,7\end{array}$ & $\begin{array}{l}4,08 \\
61\end{array}$ & $\begin{array}{l}0,16 \\
0,34\end{array}$ & $\begin{array}{l}0,22 \\
2,33\end{array}$ & $\begin{array}{l}0,28 \\
4,21\end{array}$ \\
\hline $\mathrm{SO}_{4}=$ & 0,35 & 2,65 & 4,95 & 0,02 & 0,18 & 0,34 \\
\hline $\mathrm{PO}_{4}-\mathrm{P}$ & o & 1,87 & 3,3 & 0 & 0,11 & 0,26 \\
\hline $\mathrm{NO}_{3}-\mathrm{N}$ & 125 & 242 & 365 & 5,18 & 15 & 29,4 \\
\hline $\mathrm{Ca}^{2+}$ & 7,1 & 9,1 & 11,1 & 0,40 & 0,67 & 1,01 \\
\hline $\mathrm{Mg}^{++}$ & 255 & 337 & 425 & 14,5 & 25 & 38,8 \\
\hline $\mathrm{Na}^{+}$ & 338 & 468 & 688 & 18,1 & 33,1 & 60,5 \\
\hline $\mathrm{K}^{+}$ & 140 & 356 & 710 & 6,22 & 26,26 & 59,6 \\
\hline
\end{tabular}

\begin{tabular}{|c|c|c|c|c|c|c|}
\hline St. $a_{5}$ & {[]$<$} & $\overline{[}]$ & {[]$>$} & Q.T. < & $\overline{\text { Q.T. }}$ & Q.T. > \\
\hline $\mathrm{HCO}_{3}^{-}$ & 22,9 & 25,5 & 27.9 & 2,57 & 3,52 & 4,57 \\
\hline $\mathrm{SiO}_{2}$ & 3,18 & 3,72 & 4,26 & 0,35 & 0,51 & 0,70 \\
\hline Fer total & 6 & 30 & 64 & 0,83 & 4,15 & 8,85 \\
\hline $\mathrm{so}_{4}=$ & o & 2,3 & 4,8 & o & 0,31 & 0,78 \\
\hline $\mathrm{PO}_{4}-\mathrm{P}$ & o & 1,7 & 5,5 & 0 & 0,23 & 0,90 \\
\hline $\mathrm{NO}_{3}-\mathrm{N}$ & 68 & 89 & 110 & 7,64 & 12,3 & 18 \\
\hline $\mathrm{Ca}^{3+}$ & 7,68 & 8,6 & 9,6 & 0,86 & 1,18 & 1,57 \\
\hline $\mathrm{Mg}^{++}$ & 231 & 293 & 355 & 25,9 & 40,5 & 58,2 \\
\hline $\mathrm{Na}^{+}$ & 550 & 650 & 753 & 61,7 & 89,8 & 123 \\
\hline $\mathrm{K}^{+}$ & 109 & 207 & 305 & 12,2 & 28,6 & 50 \\
\hline
\end{tabular}

\begin{tabular}{|c|c|c|c|c|c|c|}
\hline St. B & {[]$<$} & $\overline{[}]$ & {[]$>$} & Q.T. $<$ & $\overline{Q . T}$. & Q.T. > \\
\hline $\begin{array}{l}\mathrm{HCO}_{3}^{-} \\
\mathrm{SiO}_{2} \\
\mathrm{SO}_{4}= \\
\mathrm{PO}_{4}-\mathrm{P} \\
\mathrm{NO}_{3}{ }^{-\mathrm{N}} \\
\mathrm{Ca}^{++} \\
\mathrm{Mg}^{++} \\
\mathrm{Na}^{+} \\
\mathrm{K}^{+}\end{array}$ & $\begin{array}{c}51 \\
2,32 \\
4,25 \\
1,1 \\
117 \\
18,6 \\
246 \\
336 \\
225\end{array}$ & $\begin{array}{c}53,8 \\
2,48 \\
5,9 \\
2,4 \\
163 \\
20,4 \\
286 \\
453 \\
425\end{array}$ & $\begin{array}{c}56,5 \\
2,57 \\
7,6 \\
3,7 \\
210 \\
22,2 \\
326 \\
571 \\
625\end{array}$ & $\begin{array}{c}57,8 \\
2,61 \\
4,66 \\
1,21 \\
128 \\
21,2 \\
280 \\
400 \\
247\end{array}$ & $\begin{array}{c}67,3 \\
3,10 \\
7,39 \\
3 \\
204 \\
25,5 \\
358 \\
567 \\
532\end{array}$ & $\begin{array}{c}78,2 \\
3,67 \\
1,68 \\
5,27 \\
299 \\
30,5 \\
450 \\
779 \\
891\end{array}$ \\
\hline
\end{tabular}

\begin{tabular}{|c|c|c|c|c|c|c|}
\hline st. $a_{2}$ & {[]$<$} & $\overline{[\bar{c}}$ & {[]$>$} & Q.T. < & $\overline{\mathbf{Q . T}}$ & Q.T. > \\
\hline $\mathrm{HCO}_{3}^{-}$ & 10.9 & 13,2 & 14,6 & 0,06 & 1,13 & 1,63 \\
\hline $\mathrm{SiO}_{2}$ & 1,91 & 2,48 & 3,05 & 0,12 & 0,22 & 0,35 \\
\hline Fer total & o & 8 & 58 & o & 0,69 & 5 \\
\hline $\mathrm{SO}_{4}=$ & 0,2 & 2,1 & 4 & 0,017 & 0,17 & 0,44 \\
\hline $\mathrm{PO}_{4}-\mathrm{P}$ & 0.2 & 1,9 & 3,8 & 0,02 & 0,164 & 0,33 \\
\hline $\mathrm{NO}_{3}-\mathrm{N}$ & 45 & 126 & 185 & 3.63 & 12,2 & 22,46 \\
\hline $\mathrm{Ca}^{7+}$ & 3,15 & 4,15 & 5,15 & 0.21 & 0,38 & 0,61 \\
\hline $\mathrm{Mg}^{++}$ & 74 & 120 & 154 & 6.04 & 12,6 & 20,22 \\
\hline $\mathrm{Na}^{+}$ & 110 & 166 & 195 & 7,86 & 16,1 & 24,1 \\
\hline $\mathrm{k}^{+}$ & 31 & 231 & 430 & 2,68 & 19,9 & 37,1 \\
\hline
\end{tabular}

\begin{tabular}{|c|c|c|c|c|c|c|}
\hline st. $\mathbf{R}$ & {[]$<$} & $\overline{[}]$ & {[]$>$} & Q.T. $<$ & $\overline{\text { Q.T. }}$ & Q.T. > \\
\hline $\mathrm{HCO}_{3}{ }^{-}$ & 42,6 & 48,2 & 53,7 & 60,4 & 70,4 & 88,3 \\
\hline $\mathrm{SiO}_{2}$ & 2,18 & 2,48 & 2,78 & 3,09 & 3,82 & 4,59 \\
\hline Fer total & 6 & 32,3 & 57 & 9,22 & 49,6 & 87,6 \\
\hline $\mathrm{SO}_{4}=$ & 2,53 & 5 & 7,43 & 3,49 & 7,68 & 12,5 \\
\hline $\mathrm{PO}_{4}^{-\mathrm{P}}$ & 0,5 & 2 & 3,5 & 0,77 & 3,07 & 5,38 \\
\hline $\mathrm{NO}_{3}^{-\mathrm{N}}$ & 66 & 159 & 252 & 91,2 & 244 & 424 \\
\hline $\mathrm{Ca}^{7+}$ & 15,9 & 17,5 & 19,2 & 22,6 & 26,9 & 31,5 \\
\hline$\Delta \mathrm{Mg}_{+}^{++}$ & 244 & 286 & 333 & 344 & 439 & 545 \\
\hline $\begin{array}{l}\mathrm{Na}^{+} \\
\mathrm{K}^{+}\end{array}$ & 295 & 430 & 565 & 440 & 661 & 913 \\
\hline $\mathrm{K}^{\prime}$ & 85 & 300 & 515 & 130 & 461 & 792 \\
\hline
\end{tabular}

\begin{tabular}{|c|c|c|c|c|c|c|}
\hline st. $a_{6}$ & {[]$<$} & $\overline{[}]$ & {[]$>$} & Q.T. < & Q.T. & Q.T. > \\
\hline $\mathrm{HCO}_{3}{ }^{-}$ & 28,3 & 30,1 & 31,9 & 5,14 & 7,81 & 10,8 \\
\hline $\mathrm{SiO}_{2}$ & 2,42 & 2,82 & 3,23 & 0,43 & 0,73 & 1,08 \\
\hline Fer total & 50 & 90 & 130 & 12,9 & 23,3 & 33,7 \\
\hline $\mathrm{SO}_{4}=$ & o & 2,56 & 5,4 & o & $\begin{array}{l}0,66 \\
0,41\end{array}$ & $\begin{array}{l}1,81 \\
0,97\end{array}$ \\
\hline $\mathrm{PO}_{4}-\mathrm{P}$ & 0,3 & 1,6 & 2,9 & ${ }_{27}^{0,054}$ & $\begin{array}{c}0,41 \\
47,4\end{array}$ & $\begin{array}{r}0,97 \\
73,1\end{array}$ \\
\hline $\begin{array}{l}\mathrm{NO}_{3}-\mathrm{N} \\
\mathrm{Ca}_{+}\end{array}$ & 149 & 183 & $\begin{array}{r}217 \\
10=5\end{array}$ & $\begin{array}{l}27 \\
1,61\end{array}$ & 2,51 & $\begin{array}{r}73,1 \\
3,53\end{array}$ \\
\hline $\mathrm{Mg}^{++}$ & 207 & 228 & $\begin{array}{l}10,3 \\
249\end{array}$ & 37,5 & 59,1 & 83,9 \\
\hline $\mathrm{Na}^{+}$ & 274 & 514 & $7^{t_{k} 6}$ & 49,7 & 133 & 251 \\
\hline $\mathrm{K}^{+}$ & 165 & 305 & 445 & 29,9 & 79 & 149 \\
\hline
\end{tabular}

\begin{tabular}{|c|c|c|c|c|c|c|}
\hline st. C & {[]$<$} & {$[\overline{]}$} & {[]$>$} & Q.T. < & Q.T. & Q.T. > \\
\hline $\mathrm{HCO}_{3}^{-}$ & 44,9 & 46,7 & 48,4 & 69,9 & 77,8 & 86 \\
\hline $\mathrm{SiO}_{2}$ & 2,36 & 2,74 & 3,11 & 3,73 & 4,56 & 5,49 \\
\hline $\begin{array}{l}\text { Fer total } \\
\text { Se }\end{array}$ & 10 & 25,7 & 41 & 16,6 & 42,8 & 68,3 \\
\hline $\mathrm{PO}_{4}-\mathrm{P}$ & $\begin{array}{l}1,7 \\
0,8\end{array}$ & $\begin{array}{l}4,55 \\
2,4\end{array}$ & 7,45 & $\begin{array}{l}2,61 \\
1,23\end{array}$ & 7,58 & 13,3 \\
\hline $\mathrm{NO}_{3}{ }_{3}-\mathrm{N}$ & 104 & 150 & 197 & 159 & 250 & 354 \\
\hline $\mathrm{Ca}^{3+}$ & 15,4 & 16,8 & 18,1 & 23,9 & 28,0 & 32,1 \\
\hline $\mathrm{Nig}^{++}$ & 243 & 282 & 320 & 381 & 470 & 566 \\
\hline $\mathrm{Na}^{+}$ & 421 & 484 & 546 & 655 & 807 & 972 \\
\hline $\mathrm{K}^{+}$ & 119 & 341 & 562 & 198 & 568 & 937 \\
\hline
\end{tabular}

\section{Lac d'Orédon}

\section{Alt. $1848 \mathrm{~m}$}

Fig. 6. - Conditions chimiques générales existant sur le réseau hydrogra phique durant la phase déneigée en 1972 , pes concentrations moyennes probables (IT) maximales $(2>$ ) et minimales $(C)$ des différents éléments $\mathrm{Ca}^{++}, \mathrm{HCO}_{3}^{-}, \mathrm{SO}_{+}{ }^{\circ}, \mathrm{SiO}_{2}$ en $\mathrm{mg} / 1$; autres éléments en $\mu \mathrm{g} / \mathbf{1} ; 2$ ) les quantités transportées probables (Q.T.), maximales (Q.T. $>$ ) et minimales
$(Q . T .<)-\mathrm{Ca}^{++}, \mathrm{HCO}_{3}^{-}, \mathrm{SO}_{4}{ }^{*}$ et $\mathrm{SiO}_{2}$ en $\mathrm{t}$; autres éléments en kg. 